\title{
Study of Third-Grade Fluid under the Fuzzy Environment with Couette and Poiseuille Flows
}

\author{
Muhammad Nadeem, ${ }^{1}$ Imran Siddique, ${ }^{1}$ Rifaqat Ali ${ }^{D},{ }^{2}$ Nawa Alshammari $\left(\mathbb{D},{ }^{3}\right.$ \\ Raja Noshad Jamil $\mathbb{D},{ }^{1}$ Nawaf Hamadneh $\mathbb{D},{ }^{3}$ Ilyas Khan $\mathbb{D}^{4},{ }^{4}$ and Mulugeta Andualem ${ }^{5}{ }^{5}$ \\ ${ }^{1}$ Department of Mathematics, University of Management and Technology, Lahore 54770, Pakistan \\ ${ }^{2}$ Department of Mathematics, College of Science and Arts, King Khalid University, Muhayil, Abha 61413, Saudi Arabia \\ ${ }^{3}$ Department of Basic Sciences, College of Science and Theoretical Studies, Saudi Electronic University, \\ Riyadh 11673, Saudi Arabia \\ ${ }^{4}$ Department of Mathematics, College of Science Al-Zulf, Majmaah University, Al Majma'ah 11952, Saudi Arabia \\ ${ }^{5}$ Department of Mathematics, Bonga University, Bonga, Ethiopia
}

Correspondence should be addressed to Nawa Alshammari; n.alshammari@seu.edu.sa, Ilyas Khan; i.said@mu.edu.sa, and Mulugeta Andualem; mulugetaandualem4@gmail.com

Received 3 September 2021; Revised 1 November 2021; Accepted 20 November 2021; Published 17 January 2022

Academic Editor: Arunava Majumder

Copyright (C) 2022 Muhammad Nadeem et al. This is an open access article distributed under the Creative Commons Attribution License, which permits unrestricted use, distribution, and reproduction in any medium, provided the original work is properly cited.

\begin{abstract}
In this work, fundamental flow problems, namely, Couette flow, fully developed plane Poiseuille flow, and plane Couette-Poiseuille flow of a third-grade non-Newtonian fluid between two horizontal parallel plates separated by a finite distance in a fuzzy environment are considered. The governing nonlinear differential equations (DEs) are converted into fuzzy differential equations (FDEs) and explain our approach with the help of the membership function (MF) of triangular fuzzy numbers (TFNs). Adomian decomposition method (ADM) is used to solve fundamental flow problems based on FDEs. In a crisp environment, the current findings are in good accord with their previous numerical and analytical results. Finally, the effect of the $\alpha$-cut $(\alpha \in[0,1])$ and other engineering constants on fuzzy velocity profile are invested in graphically and tabular forms. Also, the variability of the uncertainty is studied through the triangular MF.
\end{abstract}

\section{Introduction}

The non-Newtonian fluids have gained considerable attention from scientists because of extensive applications in engineering, science, and industry. Various industrial ingredients fall into this bunch, such as biological solutions, soap, paints, cosmetics, tars, shampoos, mayonnaise, blood, yoghurt, syrups, and glues, etc. Due to the intricate nature of non-Newtonian fluids, it is very hard to establish a single model that can describe the characteristics of all nonNewtonian fluids. So, the fluids of differential type [1] have received superior consideration by researchers. Here, we will consider the third-grade fluids (differential type by a subclass), which have been studied effectively in numerous types of flow mechanisms [2-9]. In fluid dynamics, the study of three fundamental flows specifically (Couette, Poiseuille, and generalized Couette flow) attracts the researchers by various non-Newtonian fluids, due to their uses in science, engineering, and industry. The unidirectional flows are used in polymer engineering, for instance, die flow, injection moulding, extrusion, plastic forming, continuous casting, and asthenosphere flows [10-13]. ADM was introduced by Adomian [14-16]. ADM is a reliable, effective, and powerful technique to calculate linear and nonlinear DEs. It gives analytical solutions in the form of an infinite convergent series The ADM has various imperative points of interest over other scientific techniques just as mathematical strategies, no linearization, discretization, perturbation, and spatial transformation. Siddiqui et al., [17] deliberated parallel plate flow of a third-grade fluid using ADM and 
compare the results with numerical technique." Pirzada and Vakaskar [18] calculated the solution of the fuzzy heat equation with the help of fuzzy ADM. Paripour et al. [19] studied the analytical solution of hybrid FDEs by using the fuzzy ADM and predictor-corrector method, which shows $\mathrm{ADM}$ is better than the predictor-corrector method. "Also, Siddiqui et al., [20] provided a comparison of ADM and Homotopy perturbation method (HPM) in terms of squeezing flow between two circular plates. Their analysis shows that ADM is better than HPM. In the review of literature, third-grade fluid problems are studied only for crisp cases."

Fluid flow plays the main role in the field of science and engineering. The rise is in an extensive range of problems like chemical diffusion, magnetic effect, and heat transfer, etc. After governing these physical problems convert into linear or nonlinear DEs. In general, the physical problems with involved geometry, coefficients, parameters, initial and boundary conditions greatly affect the solution of DEs. Then the coefficients, parameters, initial and boundary conditions are not crisp due to the mechanical defects, experimental and measurement errors, etc. So in this situation, fuzzy sets theory (FST) is an effective tool for a better understanding of the considered phenomena and it is more accurate than assuming the crisp physical problems. More precisely, the FDEs play a major role to reduce the uncertainty and proper way to describe the physical problem which arises in uncertain parameters, initial and boundary conditions.

Zadeh [21] established the FST in 1965. FST is an extremely useful technique for defining situations in which information is ambiguous, hazy, or uncertain. FST is entirely defined by its MF or sense of belonging. In FST, the MF allocates a number between 0 and 1 to each element in the discourse universe. On the other hand, the degree of nonbelongingness is a complement to "one" of the membership degree. The fuzzy number (FN) is a generalization of the classical real number (which uses absolute 0 and 1 only, and nothing in between) with additional properties. FN can be expected as a function whose domain is specified zero to one. This domain is called an MF. Every numerical value in the domain is allocated a definite grade of MF where 0 signifies the minimum possible grade and 1 is the maximum possible grade. FNs are competent in modelling epistemic uncertainty and its circulation. FNs are a very useful tool for FDEs, fuzzy analysis and other applications in fuzzy logic. Arithmetic operations on FNs were developed by Dubois and Prade [22]. The impreciseness or vagueness can be defined mathematically using FNs. The information of dynamical systems modelled by ODEs or PEDs is commonly incomplete, vague, or uncertain, while FDEs represent a proper way to model the dynamical systems under vagueness or uncertainty. Seikala [23] introduced the fuzzy differentiability concept. Later on, Kaleva [24] presented fuzzy differentiation and integration. Kandel and Byatt [25] introduced the FDEs in 1987. Buckley et al. [26] used two methods extension principle and FNs for the solution of FDEs. Nieto [27] studied the Cauchy problem for continuous FDEs. Lakshmikantham and Mohapatra studied the initial value problems for FDEs that have been commenced in [28]. Park and Han [29] used successive approximation methods for the existence and uniqueness solution of FDE. Hashemi et al. [30] used HAM (Homotopyianalysis method) to calculate the analytical solutions for the system of fuzzy differential equations (SFDEs). Mosleh [31] used universal approximation and fuzzy neural network methods to solve the SFDEs. Gasilov et al. [32] developed the geometric method to solve SFDEs. Khastan and Nieto [33] used a generalized differentiability concept to solve the secondorder FDE. A few decades ago, there have been many studies revolving around the concept of FDEs. [34-47] Biswal et al. [48] studied the Natural convection of nanofluid flow between two parallel plates using HPM in a fuzzy environment. The volume fraction of nanoparticles is considered as TFN and also shows the fuzzy result is better than a crisp result. Borah et al. [49] discussed the MHD flow of second-grade fluids in a fuzzy environment using fractional derivatives Atangana-Baleanu and Caputo-Fabrizio. The nondimensional governing equations convert into fuzzified governing equations with the help of the Zadeh extension principle and triangular fuzzy number. MHD and ohmic heating on the third-grade fluid in an inclined channel in a fuzzy environment was investigated by Nadeem et al. [50]. To discuss the uncertainty, the triangle membership function was used, as shown in Figure 1. Furthermore, FST has been employed by several researchers to accomplish well-known scientific and engineering conclusions [51-59].

The above-mentioned works motivated us to develop a model to describe the fuzzyluncertain analysis for fundamental flow problems, namely, plane Couette, fully developed plane Poiseuille, and plane Couette-Poiseuille flow of the third-grade fluid between two parallel plates. The basic purpose of this article is to show the uncertain flow mechanism through FDEs and the generalization to the work of Siddiqui et al. [17] in the circumstance of fuzzy environment.

The article is structured as follows. Basic preliminaries are given in Section 2. In Section 3, the governing equations of the proposed study and changed governing equations in the fuzzy form for solving by a fuzzy ADM are developed. Results and discussion in graphical and tabular forms are presented in Section 4. In Section 5, some conclusions are given.

\section{Preliminaries about FST}

Definition 1 (see $[21,50]$ ). Fuzzy set $\bar{Z}$ is defined as a set of ordered pairs such that $\bar{Z}=\left\{x, \mu_{\bar{Z}}(x): x \in U, \mu_{\bar{Z}}(x) \in\right.$ $[0,1]\}$; here $U$ is the universal set, $\mu_{\bar{Z}}(x)$ is $\mathrm{MF}$ of $\bar{Z}$ and mapping defined as $\mu_{\bar{Z}}(x): U \longrightarrow[0,1]$.

Definition 2 (see $[22,50]$ ). $\alpha$ - cut or $\alpha$ - level of a fuzzy set $\bar{Z}$, defined by $0 Z_{\alpha}=\left\{x / \mu_{\bar{Z}}(x) \geq \alpha\right\}$, where $Z_{\alpha}$ is crisp set and $0 \leq \alpha \leq 1$.

Definition 3 (see $[22,50])$. Let $\bar{Z}=(\delta, \chi, \eta)$ with $\operatorname{MF} \mu_{\bar{Z}}(x)$ called a TFNs if 


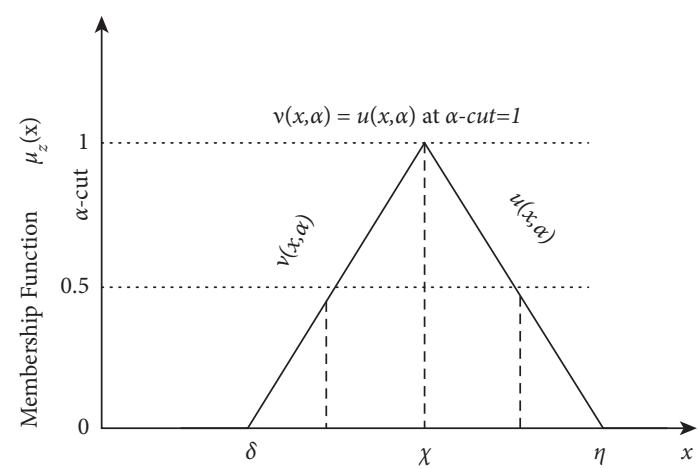

FIgURE 1: Triangular membership function.

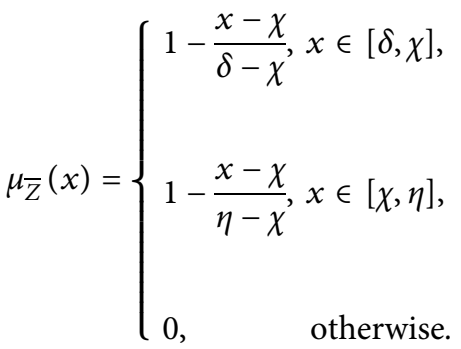

The TFNs with peak (center) $\chi$, right width $\eta-\chi>0$, left width $\chi-\delta>0$, and representation of ordered pair functions through $\alpha$-cut approach is written as $\bar{Z}=[v(x ; \alpha)$, $u(x ; \alpha)]=[\delta+\alpha(\chi-\delta), \eta-\alpha(\eta-\chi)]$, where $0 \leq \alpha \leq 1$. TFNs satisfy the following conditions: (i) $v(x ; \alpha)$ is nondecreasing on $[0,1]$. (ii) $u(x ; \alpha)$ is nonincreasing on $[0,1]$. (iii) $u(x ; \alpha)=v(x ; \alpha)$ on $[0,1]$. (iv) $v(x ; \alpha)$ and $u(x ; \alpha)$ are bounded on left continuous and right continuous at $[0,1]$, respectively. (v) If $v(x ; \alpha)=u(x ; \alpha)$ then it is called a crisp solution.

Definition 4 (see $[23,50]$ ). Let $I^{\mp}$ be an interval such that $I^{\mp} \subseteq R$. A mapping $\bar{u}: I^{\mp} \longrightarrow F^{*}$ is called a fuzzy process, defined as $\bar{u}(x ; \alpha)=[v(x ; \alpha), u(x ; \alpha)], x \in I^{\mp}$ and $0 \leq \alpha \leq 1$. The derivative $\mathrm{d} \bar{u}(x ; \alpha) / \mathrm{d} x \in F^{*}$ of a fuzzy process $\bar{u}(x ; \alpha)$ is defined as $\mathrm{d} \bar{u}(x ; \alpha) / \mathrm{d} x=[(\mathrm{d} v(x ; \alpha) / \mathrm{d} x),(\mathrm{d} u(x ; \alpha) / \mathrm{d} x)]$.

Definition 5 (see $[23,50])$. Let $I^{\mp} \subseteq R$ and $\bar{u}(x ; \alpha)$ be a fuzzy valued function define on $I^{\mp}$. Let $\bar{u}(x ; \alpha)=[v(x ; \alpha), u(x ; \alpha)]$ for all $\alpha$-cut. Assume that $v(x ; \alpha)$ and $u(x ; \alpha)$ have continuous derivatives or differentiable, for all $x \in I^{\mp}$ and $0 \leq \alpha \leq 1 \quad$ then $\quad[\mathrm{d} \bar{u}(x ; \alpha) / \mathrm{d} x]_{\alpha}=[(\mathrm{d} v(x ; \alpha) / \mathrm{d} x)$, $(\mathrm{d} u(x ; \alpha) / \mathrm{d} x)]_{\alpha}$. Similarly, the higher-order derivatives can be defined in the same way. Then $[\mathrm{d} \bar{u}(x ; \alpha) / \mathrm{d} x]$ satisfy the following conditions: (i) $\mathrm{d} v(x ; \alpha) / \mathrm{d} x$ is nondecreasing on $[0$, 1]. (ii) $\mathrm{d} u(x ; \alpha) / \mathrm{d} x$ is nonincreasing on $[0,1]$. (iii) $\mathrm{d} u(x ; \alpha) / \mathrm{d} x \geq \mathrm{d} v(x ; \alpha) / \mathrm{d} x$ on $[0,1]$.

\section{Basic Equations}

The basic equations which govern the flow of an incompressible fluid ignoring the thermal effects are as follows [17]:

$$
\begin{aligned}
\nabla \cdot \mathbf{V} & =0, \\
\rho \frac{\mathrm{d} \mathbf{V}}{\mathrm{d} t} & =\rho \mathbf{f}+\nabla \cdot \tau, \\
\tau & =-p_{1} \mathbf{I}+\tau_{1},
\end{aligned}
$$

where $f$ is the body force, $p_{1}$ is the pressure, $\rho$ is the density of fluid, $\mathrm{V}$ is velocity vector, $\mathrm{d} / \mathrm{d} t$ is the material derivative, $I$ is the unit tensor, $\tau$ is the stress tensor, and $\tau_{1}$ is the extra stress defined as follows:

$$
\tau_{1}=\tau_{2}+\varepsilon_{1} \tau_{3}+\kappa_{1} \tau_{4}+\kappa_{2}\left(\tau_{2} \tau_{3}+\tau_{3} \tau_{2}\right)+\kappa_{3}\left(\operatorname{tr} \tau_{2}^{2}\right) \tau_{2}
$$

Here, $\mu$ represents the coefficient of shear viscosity, $\kappa_{1}, \kappa_{2}, \kappa_{3}, \varepsilon_{1}$ and $\varepsilon_{2}$ are material constants. The tensors $\tau_{2}, \tau_{3}$ and $\tau_{4}$ are, respectively, given by the following:

$$
\begin{aligned}
& \tau_{2}=\nabla V+(\nabla V)^{T} \\
& \tau_{3}=\frac{\mathrm{d} \tau_{2}}{\mathrm{~d} t}+\tau_{2}\left[(\nabla V)^{T}+(\nabla V)\right], \\
& \tau_{4}=\frac{\mathrm{d} \tau_{3}}{\mathrm{~d} t}+\tau_{3}\left[(\nabla V)+(\nabla V)^{T}\right] .
\end{aligned}
$$

"For the problem under consideration, we assume a velocity profile for one-dimensional flow and stress tensor of the form."

$$
\begin{aligned}
\mathbf{V} & =(u(x), 0,0), \\
\tau_{1} & =\tau_{1}(x) .
\end{aligned}
$$

By utilizing equation (7), the continuity equation (2) is indistinguishably fulfilled and the equation of motion (3), without gravitational impact, becomes as follows:

$$
\begin{aligned}
\mu \frac{\mathrm{d}^{2} u}{\mathrm{~d} x^{2}}+6\left(\kappa_{2}+\kappa_{3}\right)\left(\frac{\mathrm{d} u}{\mathrm{~d} x}\right)^{2}\left(\frac{\mathrm{d}^{2} u}{\mathrm{~d} x^{2}}-\frac{\mathrm{d} p_{1}}{\mathrm{~d} y}\right) & =0, \\
-\frac{\mathrm{d} p_{1}}{\mathrm{~d} x}+\left(2 \varepsilon_{1}+\varepsilon_{2}\right)\left(\frac{\mathrm{d}}{\mathrm{d} x}\right)\left(\frac{\mathrm{d} u}{\mathrm{~d} x}\right)^{2} & =0,
\end{aligned}
$$

On introducing the modified pressure $p^{\mp}$,

$$
p^{\mp}=-p_{1}(x, y)+\left(2 \varepsilon_{1}+\varepsilon_{2}\right)\left(\frac{\mathrm{d} u}{\mathrm{~d} x}\right)^{2} .
$$

Using equation (10) in (9), we find that

$$
\frac{\mathrm{d} p^{\mp}}{\mathrm{d} x}=0 .
$$

From equation (11) $p^{\mp}=p^{\mp}(x)$ and as a result, equation (8) becomes as follows:

$$
\mu\left(\frac{\mathrm{d}^{2} u}{\mathrm{~d} x^{2}}\right)+6 \beta\left(\frac{\mathrm{d} u}{\mathrm{~d} x}\right)^{2}\left(\frac{\mathrm{d}^{2} u}{\mathrm{~d} x^{2}}-\frac{\mathrm{d} p^{\mp}}{\mathrm{d} y}\right)=0,
$$

where for simplicity we have introduced $\beta=\kappa_{2}+\kappa_{3}$.

"Equation (12) is a second-order nonlinear ordinary differential equation. This equation governs the 
unidirectional flow of a non-Newtonian third-grade fluid between two horizontal infinite parallel plates."

3.1. The Adomian Decomposition Method (ADM). In this section, we briefly outline the decomposition method [14]. To clarify the basic idea, we write the underlying nonlinear differential equation as follows:

$$
L_{1} u^{\mp}(x)+N_{1} u^{\mp}(x)=q(x),
$$

where $L_{1}$ and $N_{1}$ are linear and nonlinear operators, respectively, and $q$ is the source term.

In general, the operator $L_{1}$ can be written in the form

$$
L_{1}=\widehat{L}+R_{1}
$$

where $\widehat{L}$ is the highest order derivative in $L_{1}$ and is assumed to be easily invertible, $R_{1}$ is the remaining operator in $L_{1}$ whose order is less than the order of $\widehat{L}$.

Using equation (14) in (13), we have the following:

$$
\widehat{L} u^{\mp}(x)=q(x)-R_{1} u^{\mp}(x)-N_{1} u^{\mp}(x) .
$$

Applying $\widehat{L}^{-1}$ on the above equation, we have the following:

$$
u^{\mp}(x)=-\widehat{L}^{-1} R_{1} u^{\mp}(x)-\widehat{L}^{-1} N_{1} u^{\mp}(x)+g(x),
$$

where $g(x)$ signifies the terms arising after integration of $q(x)$ and calculate the constants of integration with the help of initial/boundary conditions. According to Adomian [14-16], $u^{\mp}(x)$ and $N_{1} u^{\mp}(x)$ can be uttered individually in the form

$$
\begin{array}{r}
u^{\mp}(x)=\sum_{n=0}^{\infty} u_{n}^{\mp}(x), \\
N_{1} u^{\mp}(x)=\sum_{n=0}^{\infty} A_{n}^{*}(x) .
\end{array}
$$

where $A_{n}^{*}, s$ are called Adomian polynomials $[14,15]$.

The algorithm of the general ADM can be communicated as follows:

$$
\begin{aligned}
u_{0}{ }^{\mp}(x) & =g(x), \\
u_{n+1}{ }^{\mp}(x) & =-\widehat{L}^{-1} A_{n}^{*}(x)-\widehat{L}^{-1} R_{1} u_{n}{ }^{\mp}(x), n \geq 0 .
\end{aligned}
$$

Thus, by calculating all components of $u^{\mp}(x)$, the solution can be written as follows:

$$
u^{\mp}(x)=\sum_{k=1}^{\infty} u_{k}^{\mp}(x) .
$$

Many researchers have established the convergence of this method [16]. In this continuation, we apply the ADM in the fuzzy sense to three flow problems $d$ problems.
3.2. Plane Couette Flow. Let us consider the steady laminar flow of an incompressible third-grade fluid between two infinite horizontal parallel plates. The lower plate is fixed while the upper plate at a distance $x=d$ is moving with unvarying speed $U$. Also assume that $x$ - axis is normal to the flow while $y$-axis is taken in the direction of flow as shown in Figure 2. In the absence of pressure gradient, the resultant differential equation (12) for such flow with boundary conditions reduces to the following:

$\mu \frac{\mathrm{d}^{2} u}{\mathrm{~d} x^{2}}+6 \beta\left(\frac{\mathrm{d} u}{\mathrm{~d} x}\right)^{2}\left(\frac{\mathrm{d}^{2} u}{\mathrm{~d} x^{2}}\right)=0$,

$$
\begin{gathered}
u(x)=0 \text { at } \quad x=0, \\
u(x)=U \text { at } \quad x=d .
\end{gathered}
$$

Introduce the following dimensionless parameters

$$
\begin{aligned}
& x^{\mp}=\frac{x}{\mathrm{~d}}, \\
& u^{\mp}=\frac{u}{U}, \\
& \beta^{\mp}=\frac{\beta U^{2}}{\mu d^{2}} .
\end{aligned}
$$

The boundary value problem (22) and (24) after dropping “ $\mp$ ” becomes

$$
\begin{array}{r}
\frac{\mathrm{d}^{2} u}{\mathrm{~d} x^{2}}+6 \beta\left(\frac{\mathrm{d} u}{\mathrm{~d} x}\right)^{2}\left(\frac{\mathrm{d}^{2} u}{\mathrm{~d} x^{2}}\right)=0, \\
u(x)=0 \text { at } \quad x=0, \\
u(x)=1 \text { at } x=1 .
\end{array}
$$

3.2.1. Solution of the Problem in Fuzzy Environment. To handle these problems, we have taken TFNs and discretization in the form of $(\delta, \chi, \eta)$ and $(d, e, f)$ for the fuzzy parameters. This discretization is used in the boundary of the parallel plates for certain flow behavior because the boundary is taken as fuzzified. In above, the governing equation (26) is taken as FDE

$$
\frac{\mathrm{d}^{2} \bar{u}(x ; \alpha)}{\mathrm{d} x^{2}}+6 \odot \beta \odot \frac{\mathrm{d}^{2} \bar{u}(x ; \alpha)}{\mathrm{d} x^{2}} \odot\left(\frac{\mathrm{d} \bar{u}(x ; \alpha)}{\mathrm{d} x}\right)^{2}=0 .
$$

Subject to fuzzy boundary conditions by using the $\alpha$ - cut approach, the fuzzy boundary conditions can be decomposed into an interval form regarding the $\alpha$ - cut. Therefore, under the $\alpha$-cut, the interval boundary conditions can be written as follows: 


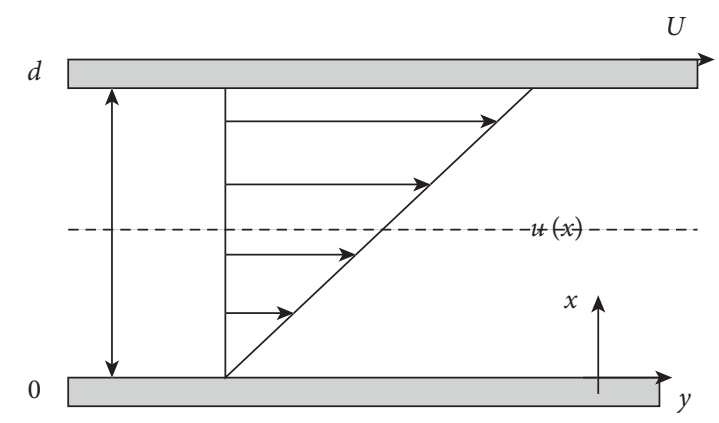

Figure 2: Geometry of the pure Couette flow.

$$
\begin{aligned}
& \bar{u}(0 ; \alpha)=[\delta+\alpha(\chi-\delta), \eta-\alpha(\eta-\chi)], \\
& \bar{u}(1 ; \alpha)=[\alpha(e-d)+d, f \alpha(f-e)], \\
& \bar{u}(x ; \alpha)=\left[\frac{\alpha-1}{100}, \frac{1-\alpha}{100}\right] \text { at } x \\
& \bar{u}(x ; \alpha)=\left[\frac{2 \alpha-2}{100}, \frac{2-2 \alpha}{100}\right] \text { at } x
\end{aligned}
$$

where operator $\odot$ defines the multiplication of fuzzy numbers with a real number and $\bar{u}(x ; \alpha)=[v(x ; \alpha), u$ $(x ; \alpha)], 0 \leq \alpha \leq 1$, is a fuzzy valued function [23]. Also $\bar{u}(x ; \alpha)$, say, is the fuzzy velocity profile, $\mathrm{d} \bar{u}(x ; \alpha) / \mathrm{d} x$ and $\mathrm{d}^{2} \bar{u}(x ; \alpha) / \mathrm{d} x^{2}$ represent the fuzzy first and second-order derivatives. Then $v(x ; \alpha)$ and $u(x ; \alpha)$ are the lower and upper bounds of fuzzy velocity profiles, respectively, while (30) are the fuzzy boundary conditions. So, equation (29) with fuzzy boundary conditions becomes

$$
\begin{gathered}
\frac{\mathrm{d}^{2} v(x ; \alpha)}{\mathrm{d} x^{2}}+6 \beta\left(\frac{\mathrm{d} v(x ; \alpha)}{\mathrm{d} x}\right)^{2} \frac{\mathrm{d}^{2} v(x ; \alpha)}{\mathrm{d} x^{2}}=0, \\
v(x ; \alpha)=\frac{\alpha-1}{100} \text { at } \quad x=0, \\
v(x ; \alpha)=\frac{2 \alpha-2}{100} \text { at } \quad x=1 . \\
\frac{\mathrm{d}^{2} u(x ; \alpha)}{\mathrm{d} x^{2}}+6 \beta\left(\frac{\mathrm{d} u(x ; \alpha)}{\mathrm{d} x}\right)^{2} \frac{\mathrm{d}^{2} u(x ; \alpha)}{\mathrm{d} x^{2}}=0, \\
u(x ; \alpha)=\frac{1-\alpha}{100} \text { at } \quad x=0, \\
u(x ; \alpha)=\frac{2-2 \alpha}{100} \text { at } \quad x=1 .
\end{gathered}
$$

For lower bound of velocity profile, we apply the ADM to equation (31) with the fuzzified boundary conditions (32) as follows:

$$
L_{1} v(x ; \alpha)=-6 \beta\left(\frac{\mathrm{d} v(x ; \alpha)}{\mathrm{d} x}\right)^{2}\left(\frac{\mathrm{d}^{2} v(x ; \alpha)}{\mathrm{d} x^{2}}\right),
$$

where $L_{1}=d^{2} / d x^{2}$ and inverse operator is $\widehat{L}^{-1}=\iint(\cdot) d x d x$. Applying $\widehat{L}^{-1}$ on both sides of equation (35), we have the following:

$$
v(x ; \alpha)=c_{1} x+c_{2}-6 \beta \widehat{L}^{-1}\left[\left(\frac{\mathrm{d} v(x ; \alpha)}{\mathrm{d} x}\right)^{2} \frac{\mathrm{d}^{2} v(x ; \alpha)}{\mathrm{d} x^{2}}\right],
$$

where the constants of integration are $c_{1}$ and $c_{2}$.

To solve equation (36) by the ADM, we let

$$
\begin{array}{r}
v(x ; \alpha)=\sum_{n=0}^{\infty} v_{n}(x ; \alpha), \\
N_{1} v(x ; \alpha)=\sum_{n=0}^{\infty} A_{n}^{*}(x ; \alpha),
\end{array}
$$

where

$$
N_{1} v(x ; \alpha)=\left(\frac{\mathrm{d} v(x ; \alpha)}{\mathrm{d} x}\right)^{2} \frac{\mathrm{d}^{2} v(x ; \alpha)}{\mathrm{d} x^{2}} .
$$

In view of equations (37)-(39), equation (36) provides the following:

$$
\sum_{n=0}^{\infty} v_{n}(x ; \alpha)=c_{1} x+c_{2}-6 \beta \widehat{L}^{-1}\left[A_{n}^{*}(x ; \alpha)\right] .
$$

We identify the zeroth-order component as follows:

$$
v_{0}(x ; \alpha)=c_{1} x+c_{2} .
$$

And the remaining components as the recurrence relation,

$$
v_{n+1}(x ; \alpha)=-6 \beta \widehat{L}^{-1}\left[A_{n}^{*}(x ; \alpha)\right],
$$

where $A_{n}^{*}$ are the Adomian polynomials that represent the nonlinear term in (39). The first few Adomian polynomials are as follows: 


$$
\begin{aligned}
A_{0}^{*}(x ; \alpha)= & \frac{\mathrm{d}^{2} v_{0}(x ; \alpha)}{\mathrm{d} x^{2}}\left(\frac{\mathrm{d} v_{0}(x ; \alpha)}{\mathrm{d} x}\right)^{2}, \\
A_{1}^{*}(x ; \alpha)= & \left(\frac{\mathrm{d} v_{0}(x ; \alpha)}{\mathrm{d} x}\right)^{2}\left(\frac{\mathrm{d}^{2} v_{1}(x ; \alpha)}{\mathrm{d} x^{2}}\right)+\left(2 \frac{\mathrm{d} v_{0}(x ; \alpha)}{\mathrm{d} x} \frac{\mathrm{d} v_{1}(x ; \alpha)}{\mathrm{d} x} \frac{\mathrm{d}^{2} v_{0}(x ; \alpha)}{\mathrm{d} x^{2}}\right), \\
A_{2}^{*}(x ; \alpha)= & \left(\frac{\mathrm{d} v_{1}(x ; \alpha)}{\mathrm{d} x}\right)^{2}\left(\frac{\mathrm{d}^{2} v_{0}(x ; \alpha)}{\mathrm{d} x^{2}}\right)+\left(2 \frac{\mathrm{d} v_{0}(x ; \alpha)}{\mathrm{d} x} \frac{\mathrm{d} v_{2}(x ; \alpha)}{\mathrm{d} x} \frac{\mathrm{d}^{2} v_{0}(x ; \alpha)}{\mathrm{d} x^{2}}\right) \\
& +\left(2 \frac{\mathrm{d} v_{0}(x ; \alpha)}{\mathrm{d} x} \frac{\mathrm{d} v_{1}(x ; \alpha)}{\mathrm{d} x} \frac{\mathrm{d}^{2} v_{1}(x ; \alpha)}{\mathrm{d} x^{2}}\right)+\left(\frac{\mathrm{d} v_{0}(x ; \alpha)}{\mathrm{d} x}\right)^{2}\left(\frac{\mathrm{d}^{2} v_{2}(x ; \alpha)}{\mathrm{d} x^{2}}\right) .
\end{aligned}
$$

The corresponding fuzzy boundary conditions, after using (37) in (32), become as follows:

$$
\begin{aligned}
& v_{0}(x ; \alpha)=\frac{\alpha-1}{100} \text { at } \quad x=0, \\
& v_{0}(x ; \alpha)=\frac{2 \alpha-2}{100} \text { at } \quad x=1 .
\end{aligned}
$$

And

$$
\begin{array}{ll}
v_{n}(x ; \alpha)=0 \text { at } & x=0, \\
v_{n}(x ; \alpha)=0 \text { at } & x=1, n \geq 1 .
\end{array}
$$

Solving equations (41) and (44), we obtain the zerothorder solution as follows:

$$
v_{0}(x ; \alpha)=\frac{(\alpha-1)(x+1)}{100} .
$$

Using polynomial (43) into (42), with the boundary conditions (45), we obtain the following:

$$
\begin{aligned}
v_{1}(x ; \alpha) & =v_{2}(x ; \alpha) \\
& =v_{3}(x ; \alpha)=0 \text {, an } d v_{n}(x ; \alpha)=0, n \geq 1 .
\end{aligned}
$$

Inserting equations (46) and (47) in (37), the solution of the differential equation (31) takes the form

$$
v(x ; \alpha)=\frac{1}{100}(\alpha-1)(x+1) .
$$

Similarly, the upper bound of velocity profile is as follows:

$$
u(x ; \alpha)=\frac{1}{100}(1-\alpha)(x+1) .
$$

Equations (48) and (49) represent the solutions of the fuzzy velocity profile for the flow of a fuzzified third-grade fluid between two parallel plates. In these solutions, the nonNewtonian parameter $\beta$ does not appear. Hence, solutions of fuzzy velocity profile give the same solution as for a Newtonian fluid.
3.3. Fully Developed Plane Poiseuille Flow. We consider the steady laminar flow of a third-grade fluid between two stationary infinite parallel plates under a constant pressure gradient. Assume that distance between two plates is $2 d$ and origin of the rectangular coordinates in between the plates as shown in Figure 3.

Thus, the resulting differential equation for the problem under consideration takes the form of equation (12) with the following boundary conditions $2 \mathrm{~d}$

$$
\begin{aligned}
\frac{\mathrm{d} u(x)}{\mathrm{d} x}=0 \text { at } & x=0, \\
u(x) & =0 \text { at } \quad x=d .
\end{aligned}
$$

Introducing the dimensionless parameters

$$
\begin{aligned}
& y^{\mp}=\frac{y}{d}, \\
& x^{\mp}=\frac{x}{d}, \\
& u^{\mp}=\frac{u}{U}, \\
& \beta^{\mp}=\frac{\beta U^{2}}{\mu d^{2}}, \\
& p^{\mp}=\frac{p d}{\mu U} .
\end{aligned}
$$

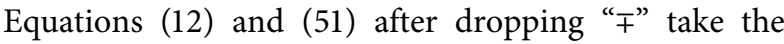
following form:

$$
\begin{gathered}
\frac{\mathrm{d}^{2} u}{\mathrm{~d} x^{2}}+\beta\left(\frac{\mathrm{d} u}{\mathrm{~d} x}\right)^{2}\left(\frac{\mathrm{d}^{2} u}{\mathrm{~d} x^{2}}-\frac{\mathrm{d} p}{\mathrm{~d} y}\right)=0, \\
\frac{\mathrm{d} u(x)}{\mathrm{d} x}=0 \text { at } \quad x=0, \\
u(x)=0 \text { at } \quad x=1 .
\end{gathered}
$$




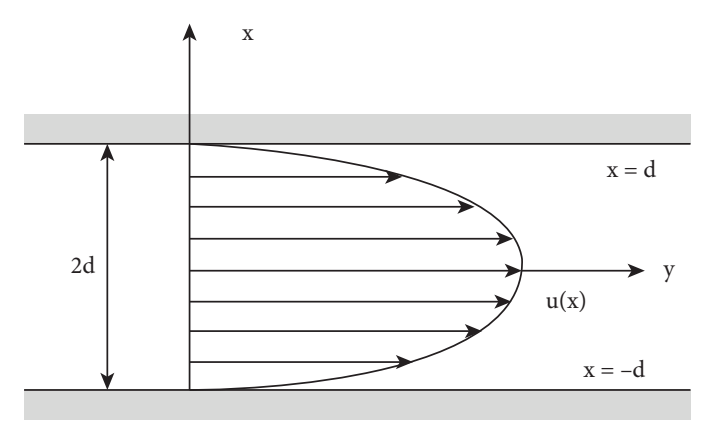

FIGURE 3: Velocity profile of the fluid flow between two parallel plates.

Now, convert equations (53) and (55) into the form of FDE as follows:

$$
\begin{aligned}
\frac{\mathrm{d}^{2} \bar{u}(x ; \alpha)}{d x^{2}}+6 \odot \beta \odot \frac{\mathrm{d}^{2} \bar{u}(x ; \alpha)}{\mathrm{d} x^{2}} \odot\left(\frac{\mathrm{d} \bar{u}(x ; \alpha)}{\mathrm{d} d x}\right)^{2}=\frac{\mathrm{d} p}{\mathrm{~d} y}, \\
\frac{\mathrm{d} \bar{u}(x ; \alpha)}{\mathrm{d} x}=\left[\frac{\alpha-1}{100}, \frac{1-\alpha}{100}\right] \text { at } \quad x=0, \\
\bar{u}(x ; \alpha)=\left[\frac{2 \alpha-2}{100}, \frac{2-2 \alpha}{100}\right] \text { at } \quad x=1 .
\end{aligned}
$$

Lower and upper bounds of velocity profile are as follows:

$$
\begin{gathered}
\frac{\mathrm{d}^{2} v(x ; \alpha)}{\mathrm{d} x^{2}}+6 \beta\left(\frac{\mathrm{d} v(x ; \alpha)}{\mathrm{d} x}\right)^{2} \frac{\mathrm{d}^{2} v(x ; \alpha)}{\mathrm{d} x^{2}}=\frac{\mathrm{d} p}{\mathrm{~d} y}, \\
\frac{\mathrm{d} v(x ; \alpha)}{\mathrm{d} x}=\frac{1}{100}(-1+\alpha) \text { at } \quad x=0, \\
v(x ; \alpha)=\frac{1}{50}(\alpha-1) \mathrm{t} \quad x=1 . \\
\frac{\mathrm{d}^{2} u(x ; \alpha)}{\mathrm{d} x^{2}}+6 \beta \frac{\mathrm{d}^{2} u(x ; \alpha)}{\mathrm{d} x^{2}}\left(\frac{\mathrm{d} u(x ; \alpha)}{\mathrm{d} x}\right)^{2}=\frac{\mathrm{d} p}{\mathrm{~d} y}, \\
\frac{\mathrm{d} u(x ; \alpha)}{d x}=\frac{1-\alpha}{100} \text { at } \quad x=0, \\
u(x ; \alpha)=\frac{1-\alpha}{50} \text { at } \quad x=1 .
\end{gathered}
$$

For lower bound of velocity profile, we apply the ADM to equation (58) as we have applied in Section 3.2.1, with the fuzzified boundary conditions (59)

$$
L_{1} v(x ; \alpha)=\frac{\mathrm{d} p}{\mathrm{~d} y}-6 \beta\left(\frac{\mathrm{d} v(x ; \alpha)}{\mathrm{d} x}\right)^{2}\left(\frac{\mathrm{d}^{2} v(x ; \alpha)}{\mathrm{d} x^{2}}\right),
$$

where $L_{1}=d^{2} / d x^{2}$. Applying the inverse operator $\widehat{L}^{-1}=\iint(\cdot) \mathrm{d} x \mathrm{~d} x$ on both sides of equation (62) yields the following:

$v(x ; \alpha)=c_{1} x+c_{2}+\widehat{L}^{-1} \frac{\mathrm{d} p}{\mathrm{~d} y}-6 \beta \widehat{L}^{-1}\left(\frac{\mathrm{d} v(x ; \alpha)}{\mathrm{d} x}\right)^{2}\left(\frac{\mathrm{d}^{2} v(x ; \alpha)}{\mathrm{d} x^{2}}\right)$,

where the constants of integration are $c_{1}$ and $c_{2}$.

Given equations (37) and (38), equation (63) is provided as follows:

$$
\sum_{n=0}^{\infty} v_{n}(x ; \alpha)=c_{1} x+c_{2}+\widehat{L}^{-1}\left(\frac{\mathrm{d} p}{\mathrm{~d} y}\right)-6 \beta \widehat{L}^{-1}\left[A_{n}^{*}(x ; \alpha)\right],
$$

Consequently, the decomposition method yields the recurrence relation,

$$
\begin{gathered}
v_{0}(x ; \alpha)=c_{1} x+c_{2}+\widehat{L}^{-1}\left(\frac{\mathrm{d} p}{\mathrm{~d} y}\right), \\
v_{n+1}(x ; \alpha)=-6 \beta \widehat{L}^{-1}\left[A_{n}^{*}(x ; \alpha)\right] .
\end{gathered}
$$

where the first few terms of the Adomian polynomial that represent the nonlinear term are defined in (43). Insight of expressions (66), we know that

$$
\begin{aligned}
& v_{1}(x ; \alpha)=-6 \beta \widehat{L}^{-1}\left[A_{0}^{*}(x ; \alpha)\right], \\
& v_{2}(x ; \alpha)=-6 \beta \widehat{L}^{-1}\left[A_{1}^{*}(x ; \alpha)\right], \\
& v_{3}(x ; \alpha)=-6 \beta \widehat{L}^{-1}\left[A_{2}^{*}(x ; \alpha)\right],
\end{aligned}
$$

The corresponding fuzzy boundary conditions become as follows:

$$
\begin{aligned}
\frac{\mathrm{d} v_{0}(x ; \alpha)}{\mathrm{d} x}=\frac{1-\alpha}{100} \text { at } & x=0, \\
v_{0}(x ; \alpha) & =\frac{\alpha-1}{50} \text { at } \quad x=1,
\end{aligned}
$$

And so on

$$
\begin{array}{r}
\frac{\mathrm{d} v_{n}(x ; \alpha)}{\mathrm{d} x}=0 \text { at } \quad x=0, \\
v_{n}(x ; \alpha)=0 \text { at } \quad x=1, n \geq 1 .
\end{array}
$$

By solving equations (65) and (66) with the fuzzified boundary conditions (68) and (69), using expression of adomian polynomials (43), equation (63) gives the solution of lower bound of velocity profile as follows: 


$$
\begin{aligned}
v(x ; \alpha)= & \frac{1}{2}\left[p x^{2}+2 F(x+1)-p\right]+\frac{\beta}{2 p}\left[E^{4}-(p x+F)^{4}+8 p F^{3}(x-1)\right]+\frac{2 \beta^{2}}{p}(p x+F)^{6} \\
& -4 \beta^{2} F^{3}(p x+F)^{3}+\frac{9 \beta^{2} E^{5} F^{3}}{p}-12 \beta^{2} F^{5}+6 \beta^{2} F^{6}+\frac{2(F E)^{3}-E^{6}}{3 p}\left(2 \beta^{2}\right) \\
& -6 \beta^{3}\left[2(p x+F)^{8}-\frac{24 p F^{3}+15 F^{3}}{10 p}(p x+F)^{5}-2 p F^{2}+x\left(12 p F^{7}-\frac{112 F^{7}}{7}\right)\right. \\
& \left.-2 p F^{6}-\frac{2 E^{8}}{p}-\frac{12 E^{5} F^{3}}{5}+\frac{112 F^{3}}{7}-12 p F^{7}-\frac{15 F^{7}}{2}\right],
\end{aligned}
$$

where $F=(\alpha-1)(p+1) / 100$ and $E=100 p+(\alpha-1)$ $(1+p) / 100$.

Equation (70) is the approximate solution of the fully developed plan Poiseuille flow and $\beta$ is a non-Newtonian parameter. By setting $\beta=0$, we have the solution for a viscous fluid.

Similarly, we can find the solution of the upper bound of velocity profile as follows:

$$
\begin{aligned}
u(x ; \alpha)= & \frac{1}{2}\left[-p+2 A_{1}(x+1)+p x^{2}\right]+\frac{\beta}{2 p}\left[A_{2}^{4}-\left(p x+A_{1}\right)^{4}+8 p A_{1}^{3}(x-1)\right]+\frac{2 \beta^{2}}{p}\left(p x+A_{1}\right)^{6} \\
& -4 \beta^{2} A_{1}{ }^{3}\left(p x+A_{1}\right)^{3}+\frac{9 \beta^{2} A_{2}^{5} A_{1}^{3}}{p}-12 \beta^{2} A_{1}{ }^{5}+6 \beta^{2} A_{1}{ }^{6}+\frac{2\left(A_{1} A_{2}\right)^{3}-A_{2}^{6}}{3 p}\left(2 \beta^{2}\right) \\
& -6 \beta^{3}\left[2\left(p x+A_{1}\right)^{8}-\frac{24 p A_{1}^{3}+15 A_{1}^{3}}{10 p}\left(p x+A_{1}\right)-2 p A_{1}{ }^{2}+x\left(12 p A_{1}{ }^{7}-\frac{112 A_{1}{ }^{7}}{7}\right)\right. \\
& \left.-2 p A_{1}{ }^{6}-\frac{2 A_{2}^{8}}{p}-\frac{12 A_{2}^{5} A_{1}{ }^{3}}{5}+\frac{112 A_{1}{ }^{3}}{7}-12 p A_{1}{ }^{7}-\frac{15 A_{1}{ }^{7}}{2}\right]
\end{aligned}
$$

where $A_{1}=(p+1)(1-\alpha) / 100$ and $A_{2}=100 p+(1-\alpha)$ $(p+1) / 100$.

3.4. Plane Couette-Poiseuille flow. Again consider the steady laminar flow of a third-grade fluid between two infinite horizontal parallel plates at a distance $d$ apart. The upper plate is moving with constant speed $U$ while the lower plate is stationary. We choose $y$-axis along with the lower plate and $x$ - axis perpendicular to it as shown in Figure 4 . The resulting differential equation in dimensionless form is (53) and the corresponding dimensionless form boundary conditions for this flow are given as follows.

$$
\begin{array}{ll}
u(x)=0 \text { at } & x=0, \\
u(x)=1 \text { at } & x=1 .
\end{array}
$$

Now we convert equations (53) and (72) into the form of FDE as follows:

$$
\begin{gathered}
\frac{\mathrm{d}^{2} \bar{u}(x ; \alpha)}{\mathrm{d} x^{2}}+6 \odot \beta \frac{\mathrm{d}^{2} \bar{u}(x ; \alpha)}{\mathrm{d} x^{2}} \\
\left(\frac{\mathrm{d} \bar{u}(x ; \alpha)}{\mathrm{d} x}\right)^{2}=\frac{\mathrm{d} p}{\mathrm{~d} y},
\end{gathered}
$$

$$
\begin{aligned}
& \bar{u}(x ; \alpha)=\left[\frac{\alpha-1}{100}, \frac{1-\alpha}{100}\right] \text { at } \quad x=0, \\
& \bar{u}(x ; \alpha)=\left[\frac{\alpha-1}{50}, \frac{1-\alpha}{50}\right] \text { at } \quad x=1 .
\end{aligned}
$$

Lower and upper bounds of velocity profiles with fuzzy boundary conditions are as follows:

$$
\begin{gathered}
\frac{\mathrm{d}^{2} v(x ; \alpha)}{\mathrm{d} x^{2}}+6 \beta \frac{\mathrm{d}^{2} v(x ; \alpha)}{\mathrm{d} x^{2}}\left(\frac{\mathrm{d} v(x ; \alpha)}{\mathrm{d} x}\right)^{2}=\frac{\mathrm{d} p}{\mathrm{~d} y}, \\
v(x ; \alpha)=\frac{\alpha-1}{100} \text { at } \quad x=0, \\
v(x ; \alpha)=\frac{\alpha-1}{50} \text { at } \quad x=1 . \\
\frac{\mathrm{d}^{2} u(x ; \alpha)}{\mathrm{d} x^{2}}+6 \beta\left(\frac{\mathrm{d} u(x ; \alpha)}{\mathrm{d} x}\right)^{2} \frac{\mathrm{d}^{2} u(x ; \alpha)}{\mathrm{d} x^{2}}=\frac{\mathrm{d} p}{\mathrm{~d} y}, \\
u(x ; \alpha)=\frac{1-\alpha}{100} \text { at } \quad x=0, \\
u(x ; \alpha)=\frac{1-\alpha}{50} \text { at } \quad x=1 .
\end{gathered}
$$

Following the same process as in previous sections and applying ADM to equation (75) with the fuzzified boundary conditions (76), we find the solution of lower bound of velocity profile as follows: 


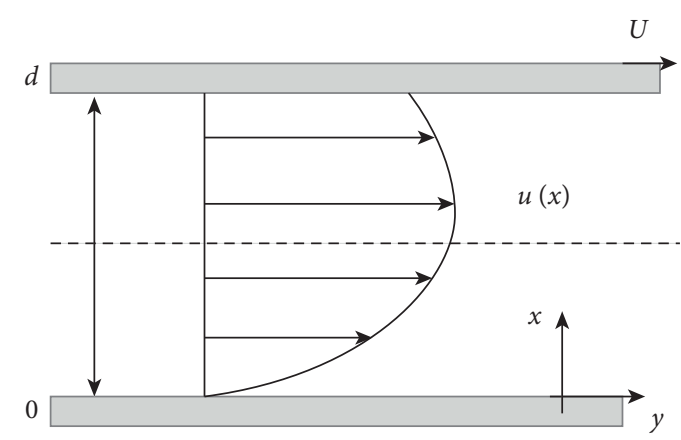

Figure 4: Geometry of the Couette-Poiseuille flow.

$$
\begin{aligned}
v(x ; \alpha)= & A(x+1)+p\left(\frac{x^{2}}{2}+D x+A\right)-p \beta\left[\frac{p^{2}(x+D)^{4}}{2}+3 A^{2} x^{2}+p(2 D+1)(x+D)^{3}-\frac{p^{2} D^{4}}{2}\right. \\
& \left.-x\left\{\frac{p^{2} D^{4}}{2}+p D^{3}(2 D+1)+3 A^{2}+\frac{p(D+1)}{2}+p(2 D+1)(D+1)^{3}\right\}-p D^{3}(2 D+1)\right] \\
& -6 \beta^{2}\left[A p^{3}\left(x^{4}+2 D x^{3}\right)+\frac{4 p^{5}(x+D)^{6}}{15}-\frac{A^{2} p^{3}(x+D)^{4}}{4}-\left(A p^{2} x^{2}+\frac{p^{3} x^{3}}{3}+D p^{3} x^{2}\right)\right. \\
& \times\left\{\frac{p^{2} D^{4}}{2}+p D^{3}(2 D+1)+3 A^{2}+\frac{p(D+1)}{2}+p(2 D+1)(D+1)^{3}\right\}-3 p A^{2} x^{2}(A+2 p D) \\
& -\frac{p^{2}(p+A)(x+D)^{4}}{2}-\frac{3 p^{4} A(x+D)^{5}}{5}-\frac{p^{2} A^{3}(x+D)^{3}}{2}+x\left\{6 p^{2} A^{2} D+\frac{p^{4}}{5}(D+1)^{5}(3 A-1)\right. \\
& \left.\left.-\frac{p^{4}}{15}(D+1)^{6}+\frac{p^{2} A}{4}(D+1)^{4}(2 p+A p+2)+\frac{p^{2} A^{3}}{2}(D+1)^{3}+3 p A^{3}-A p^{3}-2 D A p^{3}\right\}\right],
\end{aligned}
$$

where $A=\alpha-1 / 100, D=\alpha-51 / 100$. Similarly, the solution of an upper bound of the velocity profile is as follows:

$$
\begin{aligned}
u(x ; \alpha)= & B(x+1)+p\left(\frac{x^{2}}{2}+B_{1} x+B\right)-p \beta\left[\frac{p^{2}\left(x+B_{1}\right)^{4}}{2}+3 B^{2} p^{2}+p\left(2 B_{1}+1\right)\left(x+B_{1}\right)^{3}\right. \\
& -x\left\{\frac{p^{2} B_{1}^{4}}{2}+p B_{1}^{3}\left(2 B_{1}+1\right)+3 B^{2}+\frac{p\left(1+B_{1}\right)}{2}+p\left(1+2 B_{1}\right)\left(1+B_{1}\right)^{3}\right\}-\frac{p^{2} B_{1}^{4}}{2} \\
& \left.-p B_{1}^{3}\left(2 B_{1}+1\right)\right]-6 \beta^{2}\left[B p^{3}\left(x^{4}+2 B_{1} x^{3}\right)+\frac{4 p^{5}\left(x+B_{1}\right)^{6}}{15}-\frac{B^{2} p^{3}\left(x+B_{1}\right)^{4}}{4}-\left(\begin{array}{c}
p^{2} x^{2} \\
3 \\
\end{array}\right.\right. \\
& \left\{\frac{p^{2} B_{1}^{4}}{2}+p B_{1}^{3} p^{3} x^{2}\right) \\
& -3 p B^{2} x^{2}\left(B+2 p B_{1}\right)-\frac{p^{2}(p+B)\left(B_{1}+x\right)^{4}}{2}-\frac{3 p^{4} B\left(B_{1}+x\right)^{5}}{5}-\frac{p^{2} B^{3}\left(B_{1}+x\right)^{3}}{2} \\
& +x\left\{6 p^{2} B^{2} B_{1}+\frac{p^{4}}{5}\left(B_{1}+1\right)^{5}(3 B-1)-\frac{p^{4}}{15}\left(B_{1}+1\right)^{6}+\frac{p^{2} B}{4}\left(B_{1}+1\right)^{4}(2 p+B p+2)\right. \\
& \left.\left.+\frac{p^{2} B^{3}}{2}\left(B_{1}+1\right)^{3}+3 p B^{3}-B p^{3}-2 B B_{1} p^{3}\right\}\right],
\end{aligned}
$$




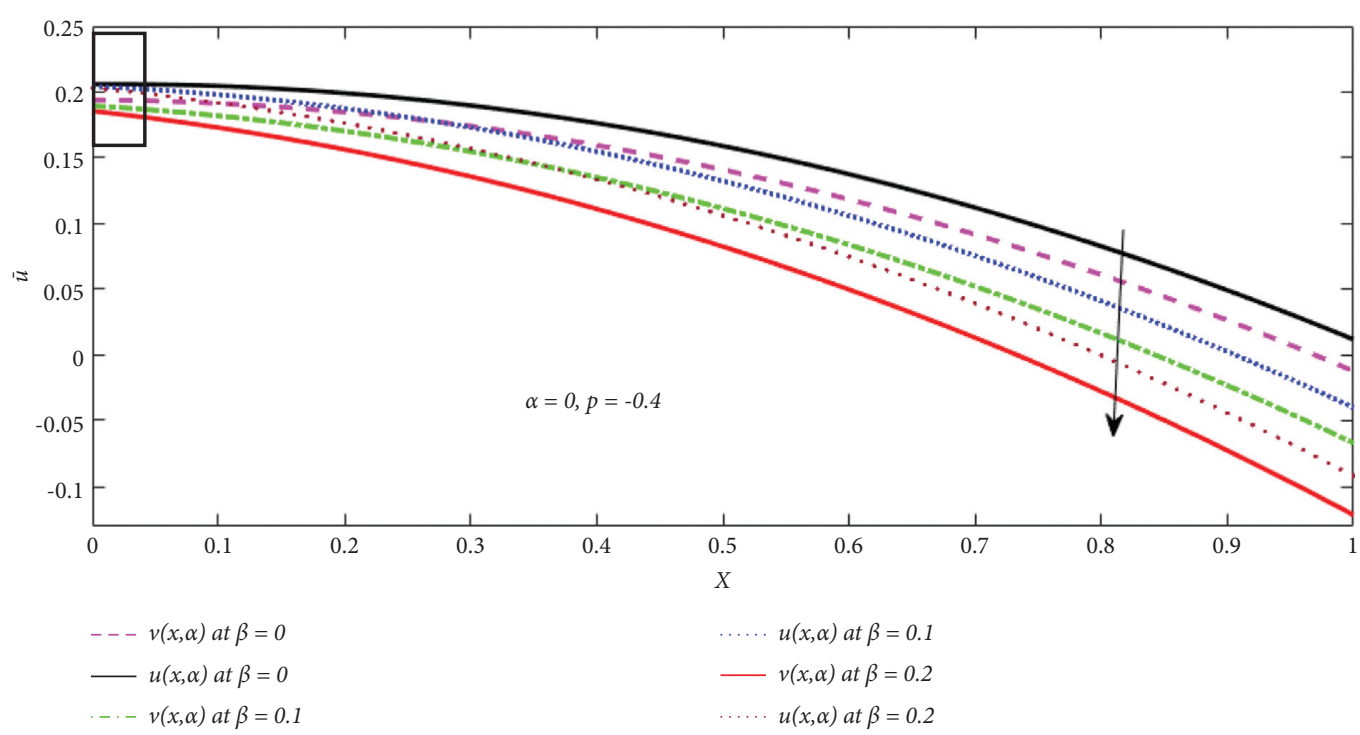

Figure 5: Fuzzy velocity profiles for influence of $\beta$.

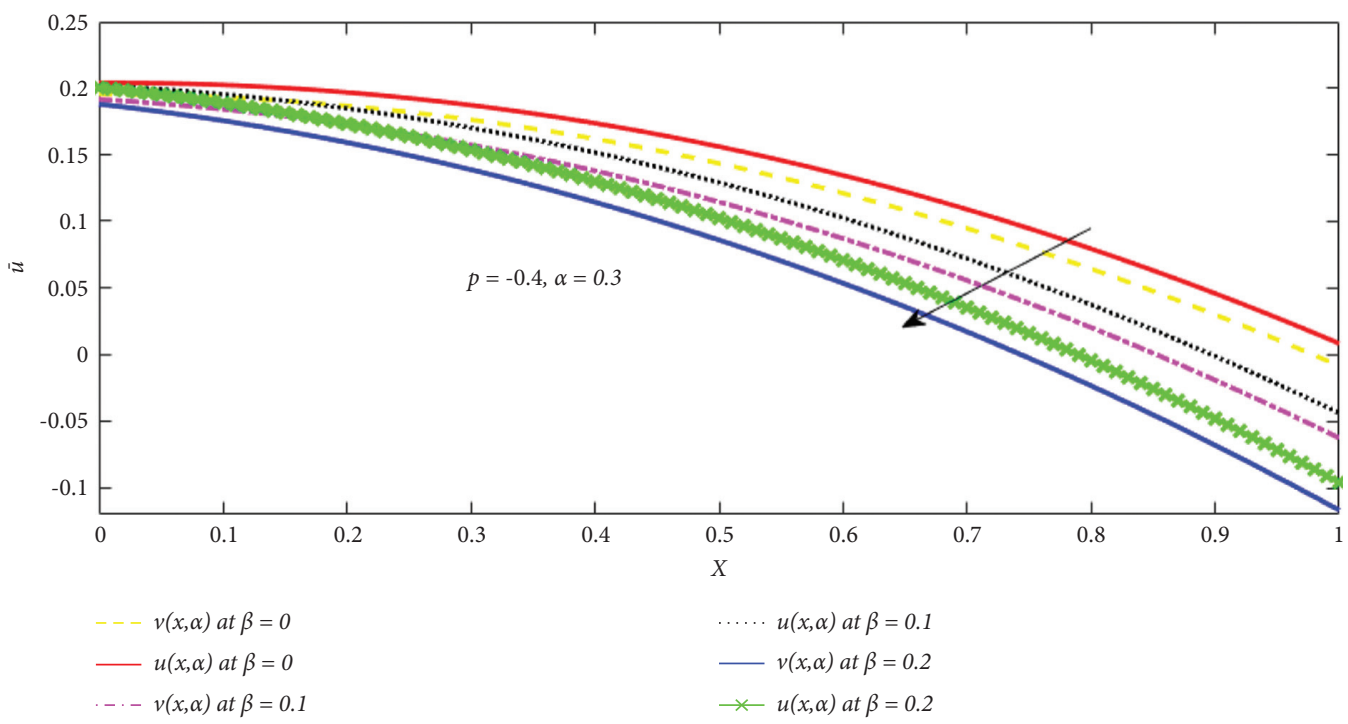

Figure 6: Fuzzy velocity profiles for influence of $\beta$.

where $B=1-\alpha / 100$ and $B_{1}=-(\alpha+49 / 100)$.

\section{Results and Discussion}

In this section, we present a numerical solution of Plane Couette flow, fully developed plane Poiseuille flow, and plane Couette-Poiseuille flow for the third-grade nonNewtonian fluid with fuzzified boundary conditions. Firstly, convert the governing equations of these problems into FDEs, then find the solutions for fuzzy velocity profiles by using ADM. Achieved fuzzy velocity profiles are plotted in Figures 5-17 for different values of $\alpha$-cut $(\alpha=0,0.3,0.7,1)$. It can be observed that as $\alpha$ increases from 0 to 1 , we have a narrow width of fuzzy velocity profiles and uncertainty decreases drastically, which finally provide crisp results for $\alpha=1$.
Tables 1-3 show the comparison of the crisp velocity profile with Siddiqui et al. [4] and Yürüsoy [9]. The validation of the present study findings was determined to be in excellent agreement.

4.1. For Plane Couette Flow. The non-Newtonian parameter $\beta$ does not exist in this solution. As a result, solutions for fuzzy velocity profiles are the same as for a Newtonian fluid.

4.2. For Fully Developed Plane Poiseuille Flow. Figures 5-8 show the effect of non-Newtonian parameter $\beta$ on the fuzzy velocity profiles with constant pressure gradient $p=d p / d y$ at different values of fuzzy parameter $\alpha$. It is observed that the lower and upper bounds of velocity profiles decrease with increasing non-Newtonian parameter 


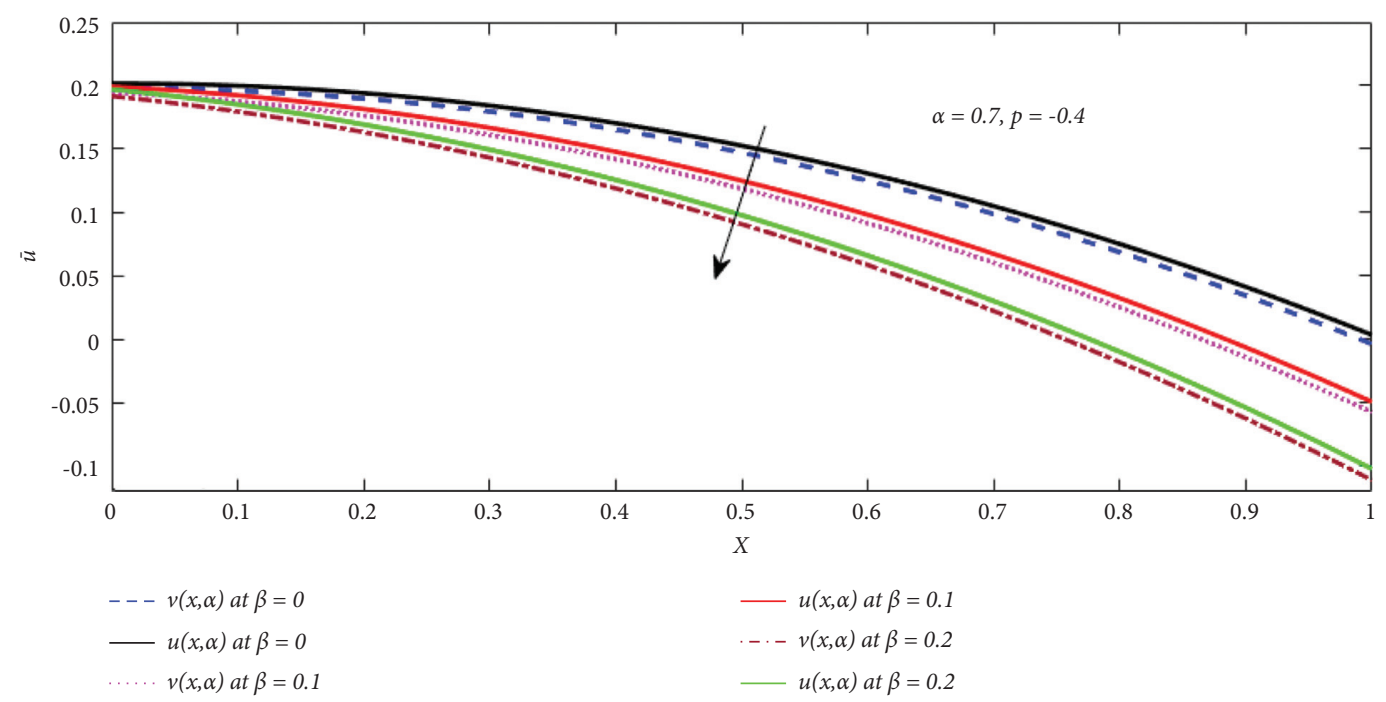

Figure 7: Fuzzy velocity profiles for influence of $\beta$.

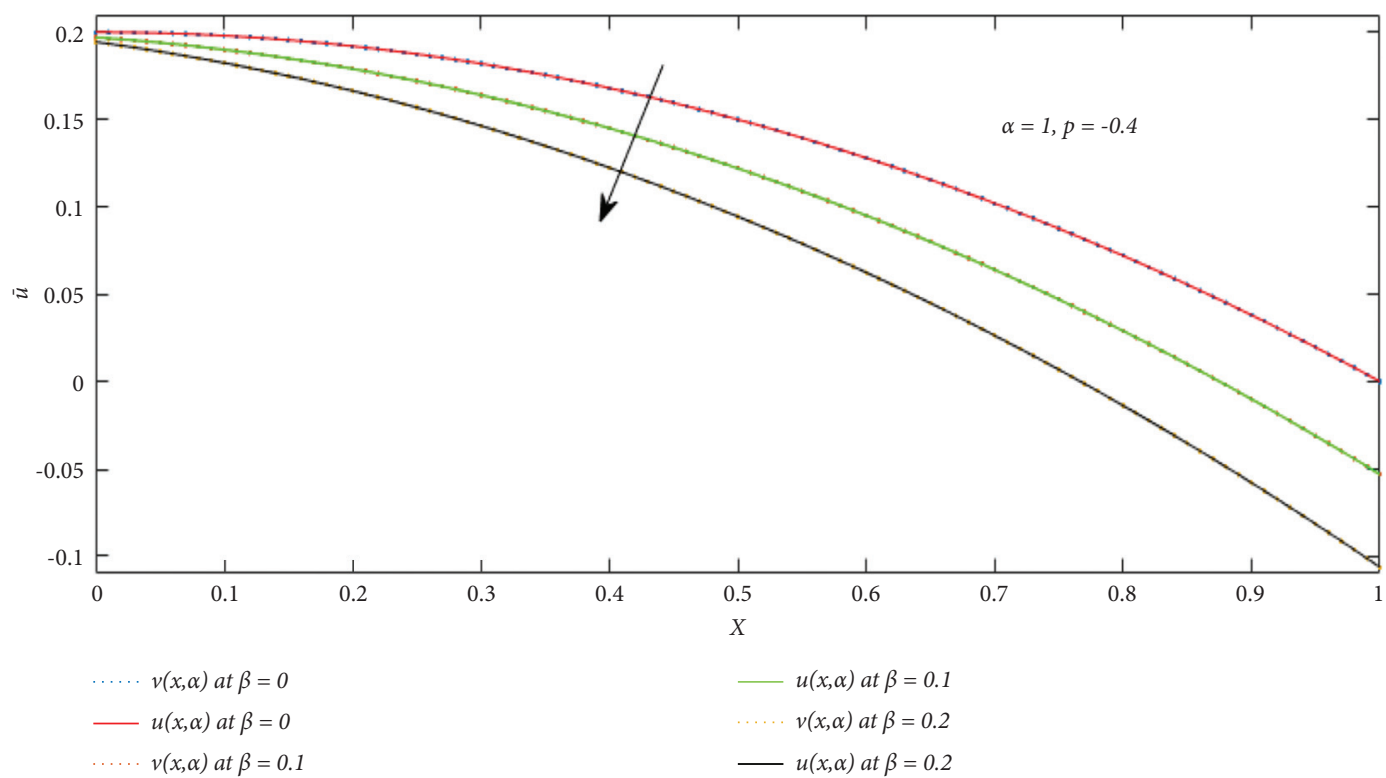

Figure 8: Fuzzy velocity profiles for influence of $\beta$.

$\beta$ as well fuzzy parameter $\alpha$. Figure 8 shows the good agreement of crisp solution or classical solution that lower and upper bounds of velocity profiles are the same at $\alpha=1$. Figure 9 describes the lower and upper bounds of fuzzy velocity profiles at the different values of $\alpha$. So, for $\alpha=1$ the fuzzy velocity profile falls into classical velocity profile, which shows the present problem is a generalization of Siddiqui et al. [17]. Figure 10 shows the uncertain behavior in terms of the triangular fuzzy plot by fixing the values of $x$ and $p=d p / d y$. The horizontal axis display the velocity profile and the vertical axis expresses the $\alpha-$ cut which range from 0 to 1 . In this figure, uncertain width gradually decreases with increasing $\alpha$-cut. We observed that $v(x ; \alpha)$ increases and $u(x ; \alpha)$ decreases with increasing of $\alpha$-cut, so the solution is strong. When $\alpha$ increases the width between lower and upper bounds of fuzzy velocity profiles decreases and when $\alpha=1$ they concur with one another. Also, the width between $v(x ; \alpha)$ and $u(x ; \alpha)$ for different values of beta is the same. This means that the uncertainty of fuzzy velocity is minimum. Table 4 shows the analysis of lower, mid and upper bounds of velocity profiles at different values of $x$ with constant pressure gradient $p=-0.4$. The mid-value of a TFN concurs with the crisp or classical value of the original problem.

4.3. For Plane Couette-Poiseuille Flow. Figures 11-14 shows the effect of non-Newtonian parameter $\beta$ on the fuzzy velocity profiles with constant pressure gradient at different values of fuzzy parameter $\alpha$. The fuzzy velocity profiles 


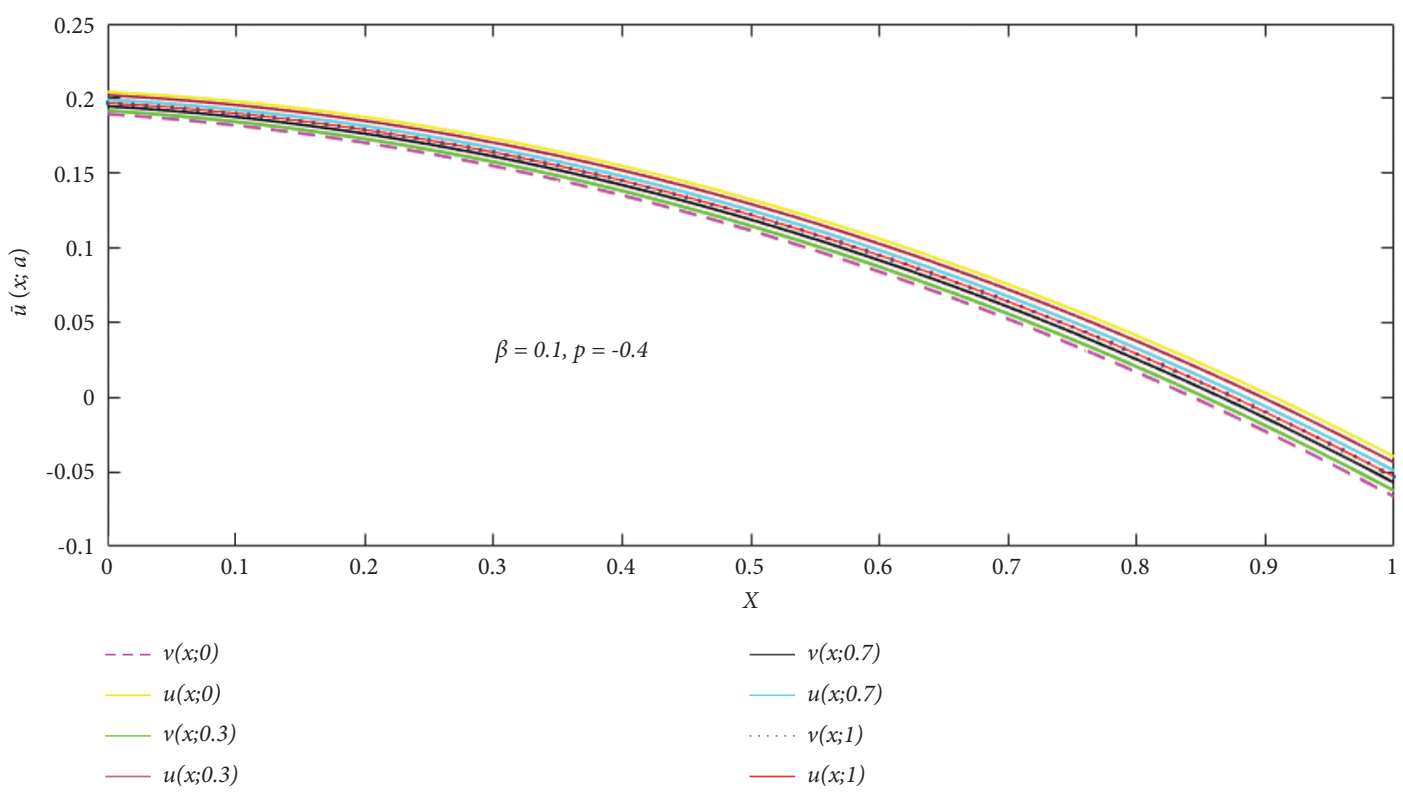

Figure 9: Fuzzy velocity profiles for different values of $\alpha$-cut $(0 \leq \alpha \leq 1)$.

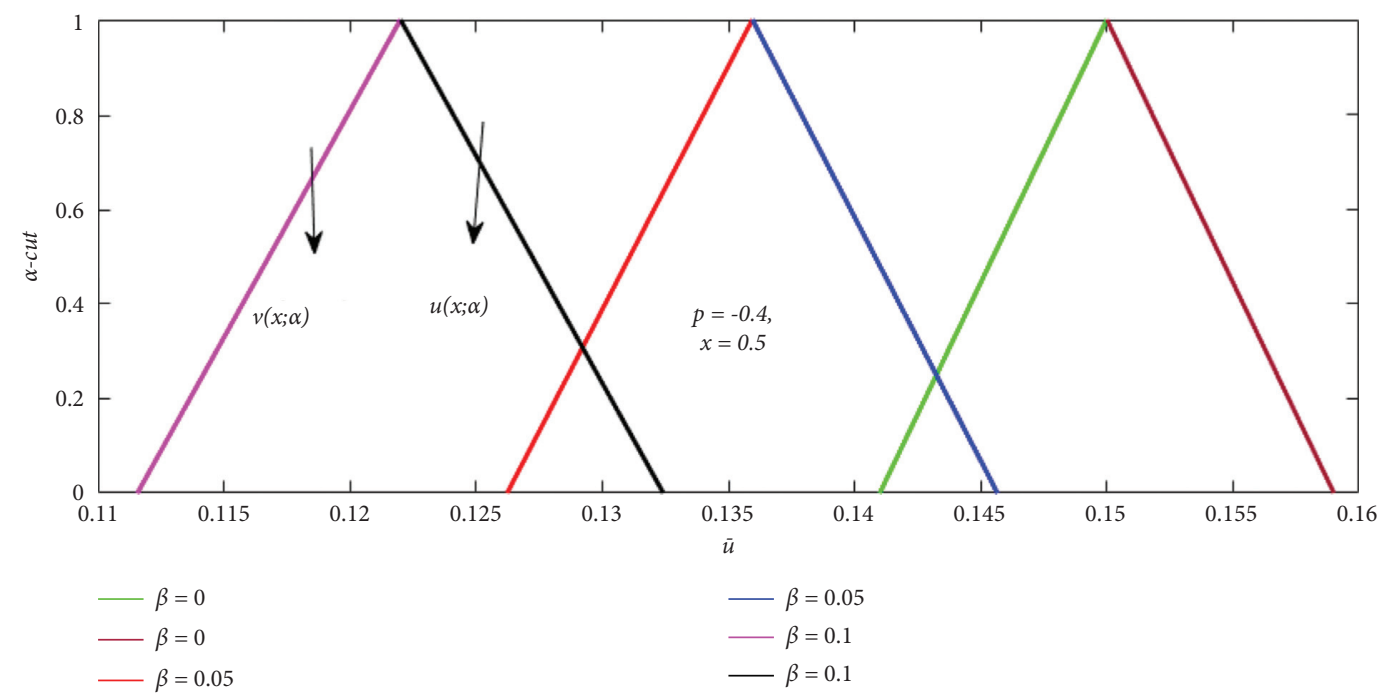

FIGURE 10: Triangular membership function of fuzzy velocity profiles for influence of $\beta$.

increases with increasing non-Newtonian parameter $\beta$ and fuzzy parameter $\alpha$. Figure 14, shows the good agreement of crisp solution or classical solution for $v(x ; \alpha)$ and $u(x ; \alpha)$ of velocity profiles at $\alpha=1$. Figure 15 describes the lower and upper bounds of fuzzy velocity profiles at the different values of $\alpha$. So, for $\alpha=1$ the fuzzy velocity profile fall into classical velocity profile, which shows the present problem is a generalization of Siddiqui et al. [17]. Figure 16 represents the fuzzy velocity profile for different ranges of the imposed pressure gradient. Figure 17 shows the uncertain behavior in terms of a triangular fuzzy plot by fixing the values of $x=0.1$ and $p=-0.6$. We observed that $v(x ; \alpha)$ increases and $u(x ; \alpha)$ decreases with increasing $\alpha$, so the solution is strong. The crisp or classical solution lies among the fuzzy solutions when $\alpha$ increases the width between lower and upper bounds of fuzzy velocity profiles decreases and at $\alpha=1$ the coherent with one another. Since the boundary conditions are fuzzy, the uncertain width gradually decreases with increasing $\alpha$ and non-Newtonian parameter $\beta$. Table 5 shows the analysis of lower, mid, and upper bounds of velocity profiles at different values of $x$ with constant pressure gradient $p=-0.6$. The mid-value of a TFN concurs with the crisp or classical value of the original problem. Furthermore, fuzzy velocity profiles always change with a certain range for any fixed $\alpha$-cut and the range gradually decreases with increasing the values of $\alpha$ - cut.

This whole discussion concludes that the fuzzy velocity profile of the fluid is a better option as compared to the crisp 


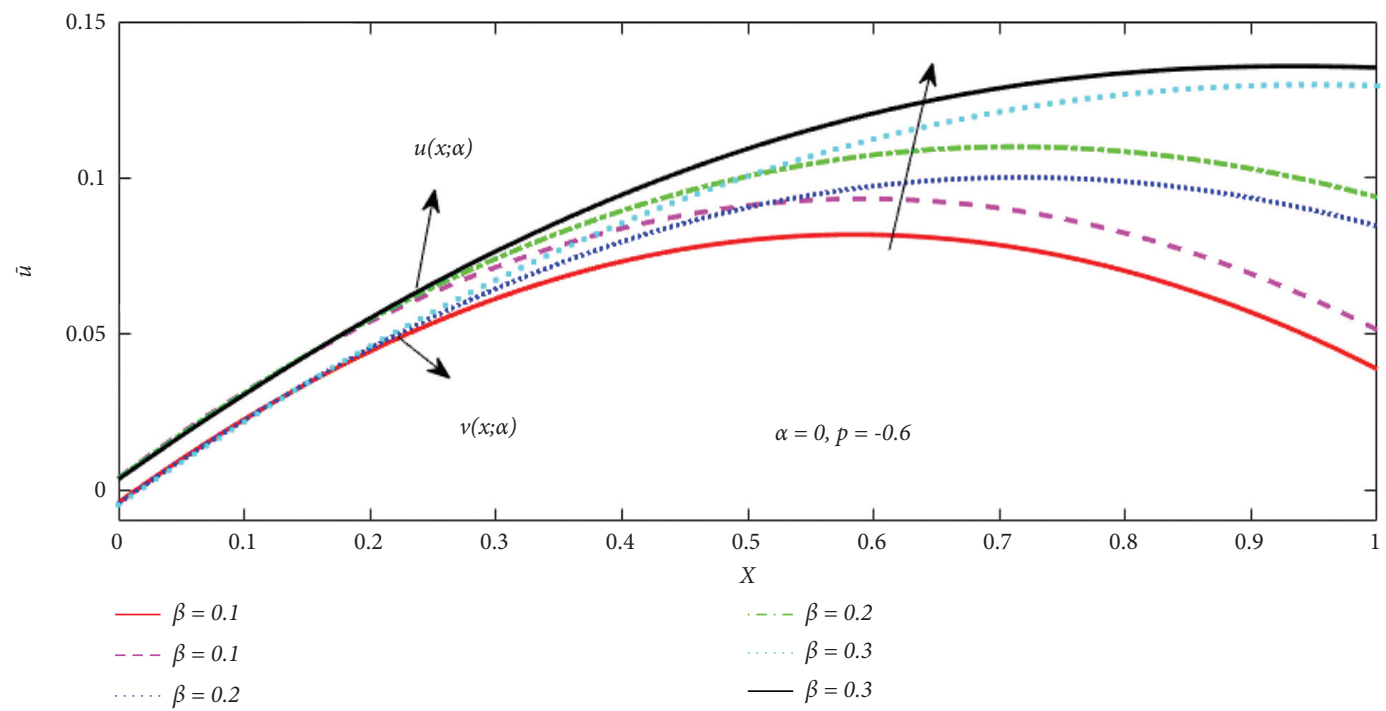

Figure 11: Fuzzy velocity profiles for influence of $\beta$.

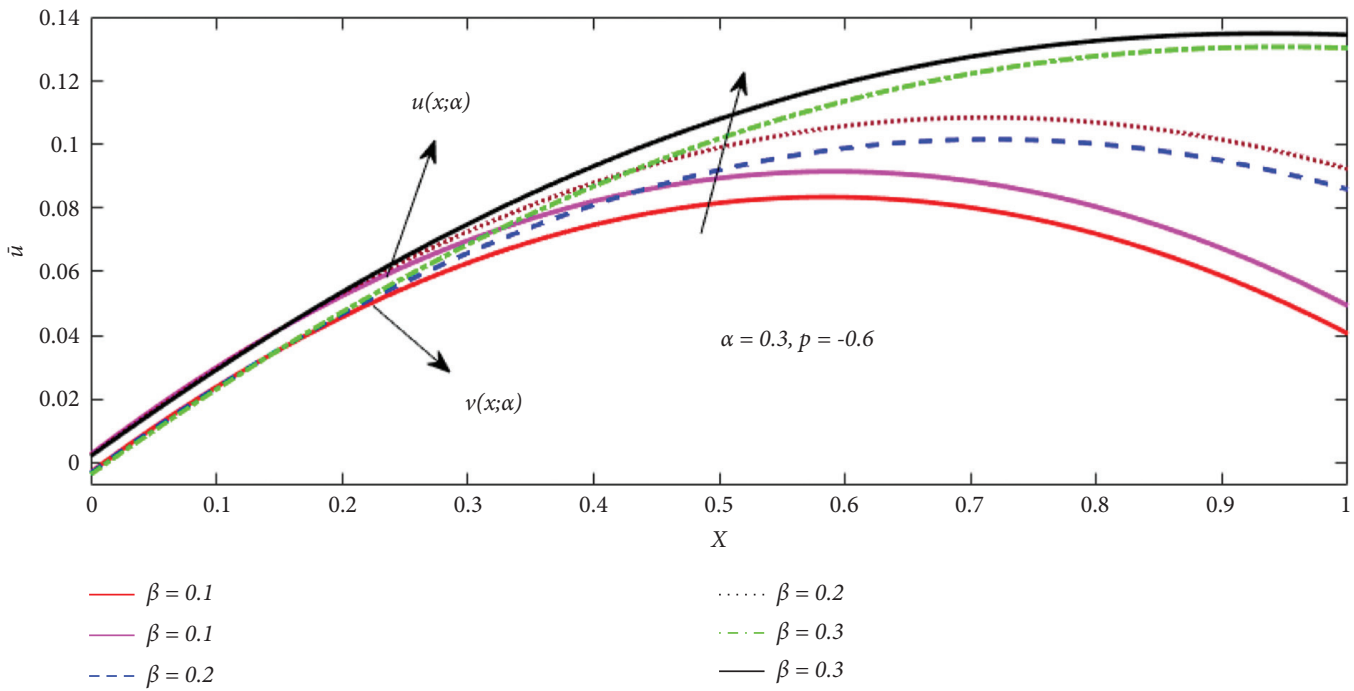

FIGURE 12: Fuzzy velocity profiles for influence of $\beta$.

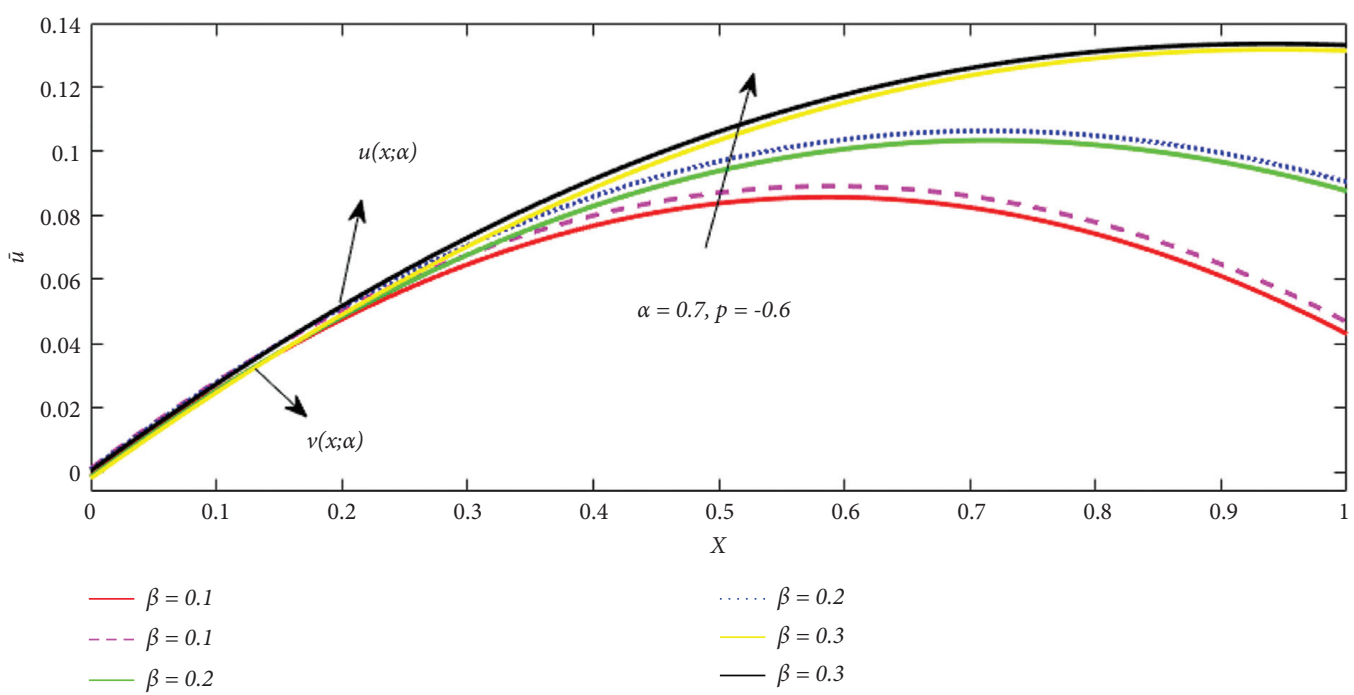

FIgURE 13: Fuzzy velocity profiles for influence of $\beta$. 


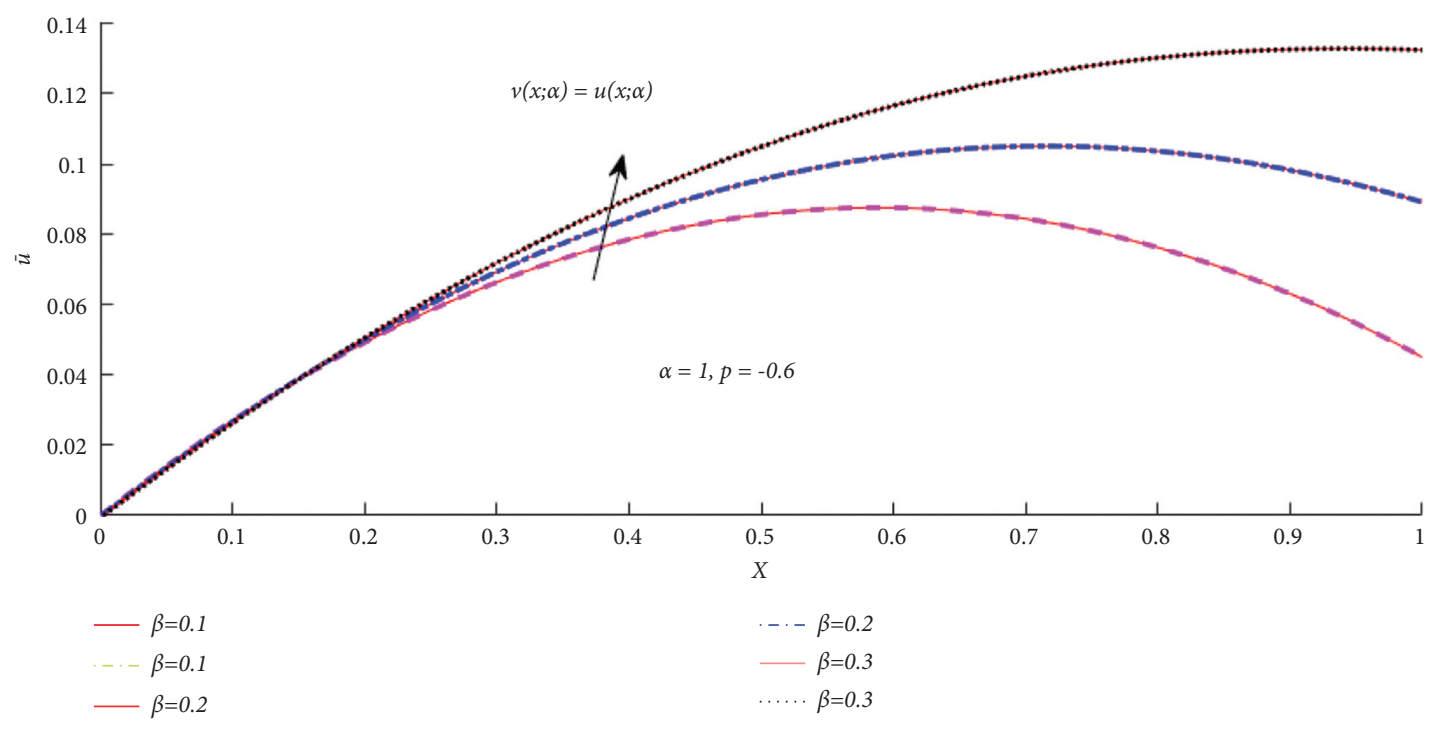

FIGURe 14: Fuzzy velocity profiles for influence of $\beta$.

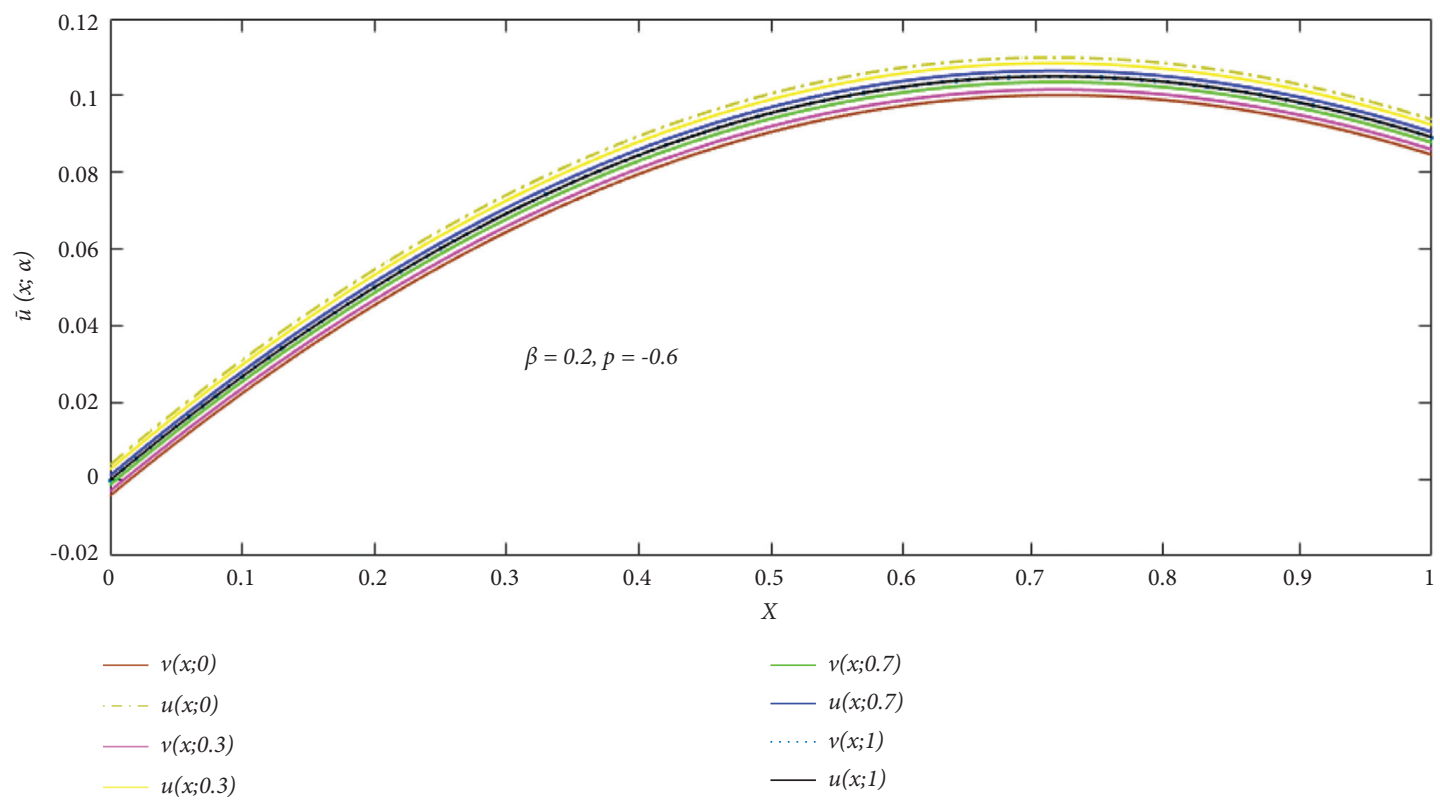

FIGURE 15: Fuzzy velocity profiles for different values of $\alpha$ - cut $(0 \leq \alpha \leq 1)$.

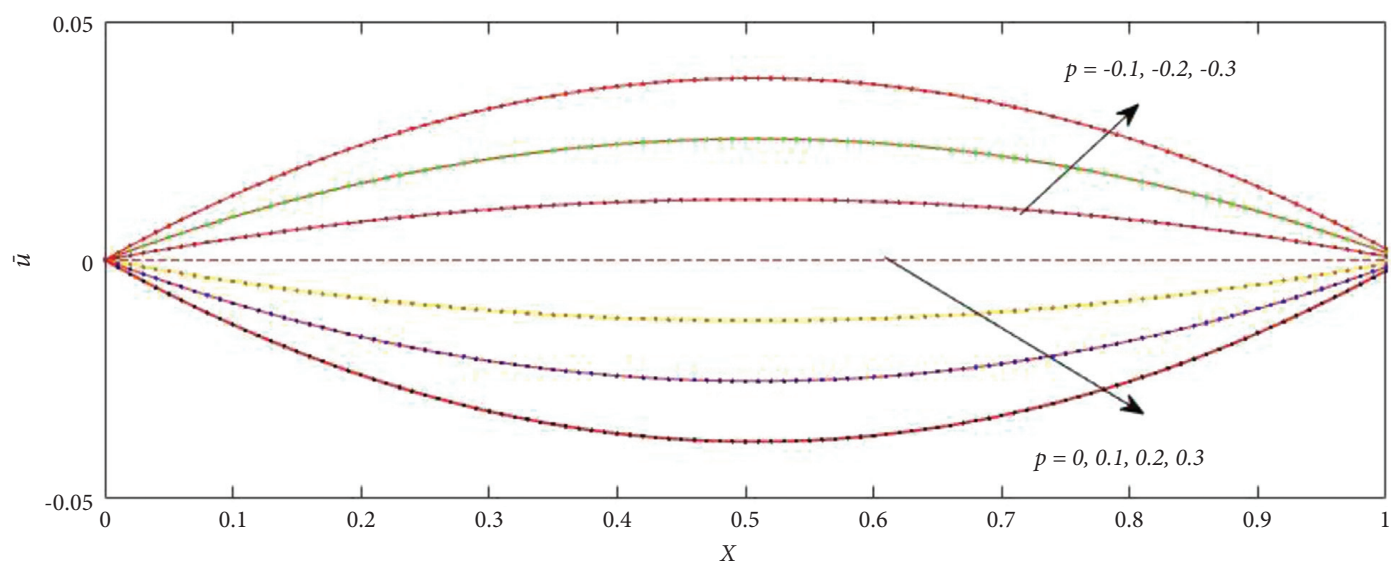

FIgURE 16: Influence of pressure gradient $\mathrm{d} p / \mathrm{d} y=p$. 


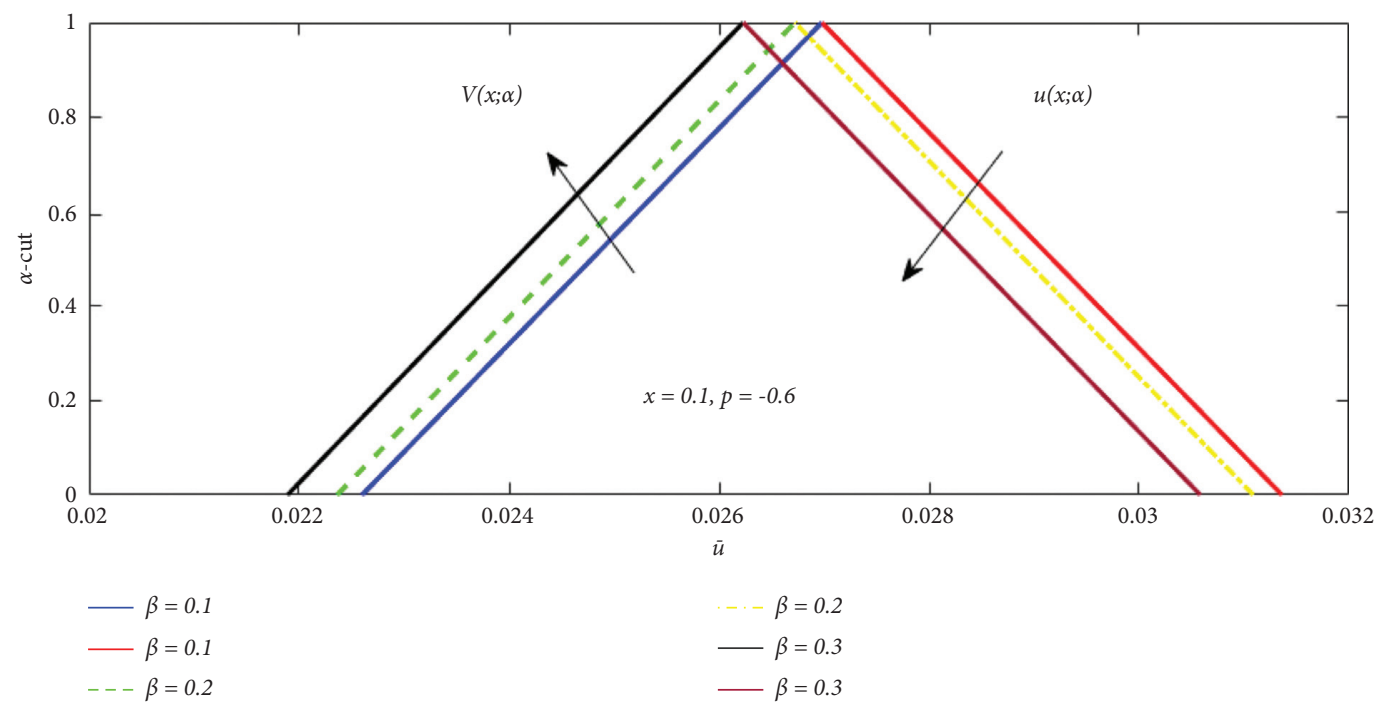

FIGURE 17: Triangular MFs of fuzzy velocity profiles for influence of $\beta$.

TABLE 1: Comparison of analytical results for the crisp velocity profile of Couette flow when $\beta=0.1, p=-0.5$, and $\alpha=1$.

\begin{tabular}{lccc}
\hline$x$ & HPM [4] & PM [9] & ADM present results \\
\hline 0 & 0 & 0 & 0 \\
0.1 & 0.0814178 & 0.091211 & 0.076017 \\
0.2 & 0.1618119 & 0.181128 & 0.151478 \\
0.3 & 0.2618251 & 0.301241 & 0.249145 \\
0.4 & 0.3410156 & 0.374915 & 0.330151 \\
0.5 & 0.4721819 & 0.467189 & 0.476182 \\
0.6 & 0.5215171 & 0.572820 & 0.518251 \\
0.7 & 0.6214168 & 0.667189 & 0.610425 \\
0.8 & 0.7514268 & 0.771810 & 0.741516 \\
0.9 & 0.8810148 & 0.882185 & 0.871816 \\
1 & 1 & 1 & 1 \\
\hline
\end{tabular}

TABLE 2: Comparison of analytical results for the crisp velocity profile of Poiseuille flow when $\beta=0.1, p=-0.5$, and $\alpha=1$.

\begin{tabular}{lccc}
\hline$x$ & HPM [4] & PM [9] & ADM present results \\
\hline 0 & 0.049141 & 0.049792 & 0.048812 \\
0.1 & 0.048818 & 0.049211 & 0.048314 \\
0.2 & 0.046718 & 0.047801 & 0.045140 \\
0.3 & 0.045168 & 0.045314 & 0.044141 \\
0.4 & 0.041015 & 0.041830 & 0.040019 \\
0.5 & 0.037180 & 0.037351 & 0.036124 \\
0.6 & 0.031417 & 0.031876 & 0.031109 \\
0.7 & 0.025001 & 0.025404 & 0.024914 \\
0.8 & 0.017912 & 0.017934 & 0.017819 \\
0.9 & 0.009214 & 0.009466 & 0.009164 \\
1 & 0 & 0 & 0 \\
\hline
\end{tabular}


TABLE 3: Comparison of analytical results for the crisp velocity profile of Couette-Poiseuille flow when $\beta=0.1, p=-0.5$, and $\alpha=1$.

\begin{tabular}{lccc}
\hline$x$ & HPM [4] & PM [9] & ADM present results \\
\hline 0 & 0 & 0 & 0 \\
0.1 & 0.081018 & 0.091401 & 0.044181 \\
0.2 & 0.121481 & 0.020762 & 0.109168 \\
0.3 & 0.194415 & 0.300141 & 0.191620 \\
0.4 & 0.281510 & 0.411359 & 0.266405 \\
0.5 & 0.380151 & 0.521415 & 0.361791 \\
0.6 & 0.481141 & 0.591618 & 0.471819 \\
0.7 & 0.601486 & 0.691619 & 0.599160 \\
0.8 & 0.721412 & 0.791819 & 0.714991 \\
0.9 & 0.881514 & 0.900410 & 0.871680 \\
1 & 1 & 1 & 1 \\
\hline
\end{tabular}

TABLE 4: Fuzzy solution of lower, mid and upper bounds of velocity profiles at different values of $x$ with constant pressure gradient $p=-0.4$.

\begin{tabular}{lccc}
\hline$x$ & $v(x ; \alpha)$ & Mid-value & $u(x ; \alpha)$ \\
\hline 0 & 0.1895776184 & 0.1970011712 & 0.2044247240 \\
0.1 & 0.1819776137 & 0.1900011710 & 0.1980247283 \\
0.2 & 0.1703775897 & 0.1790011570 & 0.1876247244 \\
0.3 & 0.1547774055 & 0.1640010163 & 0.1732246271 \\
0.4 & 0.13517655524 & 0.1450003147 & 0.1548240742 \\
0.5 & 0.11157377629 & 0.1219979280 & 0.1324220798 \\
0.6 & 0.08396651205 & 0.0949915265 & 0.1060165410 \\
0.7 & 0.05235022580 & 0.0639769107 & 0.0756035957 \\
0.8 & 0.01671756767 & 0.0289472010 & 0.0411768343 \\
0.9 & -0.0229426059 & -0.0101081214 & 0.0027263631 \\
1 & -0.0666463604 & -0.0532043200 & -0.0397622795 \\
\hline
\end{tabular}

TABLE 5: Fuzzy solution of lower, mid and upper bounds of velocity profiles at different values of $x$ with constant pressure gradient $p=-0.6$.

\begin{tabular}{lccc}
\hline$x$ & $v(x ; \alpha)$ & Mid value & $u(x ; \alpha)$ \\
\hline 0 & -0.0043186926 & -0.0002926560 & 0.0037333806 \\
0.1 & 0.0223634776 & 0.0267255366 & 0.0310875957 \\
0.2 & 0.0453558474 & 0.0499846994 & 0.0546135514 \\
0.3 & 0.0644547391 & 0.0692807804 & 0.0741068218 \\
0.4 & 0.0795257286 & 0.0844783261 & 0.0894309236 \\
0.5 & 0.0904934414 & 0.0955015114 & 0.100509581 \\
0.6 & 0.0973341525 & 0.1023287586 & 0.1073233647 \\
0.7 & 0.1000709293 & 0.1049909429 & 0.1099109565 \\
0.8 & 0.0987710557 & 0.1035731870 & 0.1083753183 \\
0.9 & 0.0935454754 & 0.0982202415 & 0.1028950076 \\
1 & 0.0845499956 & 0.0891454549 & 0.0937409143 \\
\hline
\end{tabular}

or classical velocity profile of the fluid. Crisp or classical velocity profile of fluid gives the single flow situation of the fluid while fuzzy velocity profile of fluid gives the interval flow situation like lower and upper bounds of the velocity profile.

4.3.1. Fully Developed Plane Poiseuille Flow. Fuzzy velocity profiles are provided in Figures 5-10.

4.3.2. Plane Couette-Poiseuille Flow. Fuzzy velocity profiles, influence of pressure gradient, and triangular MFs of fuzzy velocity profiles are shown in Figures 11-17, respectively.

\section{Conclusions}

In this work, we have studied the three basic fundamental flow problems in a fuzzy environment. The dimensionless nonlinear governing equations are converted into FDEs with fuzzified boundary conditions and find their solutions using ADM. For the case of a plane Couette flow, we find the same solution as in the incident of viscous fluid. For plane Poiseuille and generalized Couette flows, triangular fuzzy numbers are used for uncertainty on the dynamic behavior of fuzzy velocity profiles. The most important findings are presented below: 
(i) Fuzzy velocity profiles increases with increasing the non-Newtonian fluid parameter $\beta$ and fuzzy parameter $(\alpha \in[0,1])$.

(ii) The results are indicated that the range of calculated lower and upper-velocity profiles depends upon a fuzzy parameter.

(iii) The results are always an envelope of solutions with a crisp solution between the upper and lower bounds of the solutions. So fuzzy velocity profiles are the generalization of the crisp velocity profile for third-grade fluid between two parallel plates.

(iv) Furthermore, it is observed that, in triangular MFs, if the width of fuzzy or uncertain velocity becomes more, then the boundary conditions are more sensitive, while for less width of fuzzy or uncertain velocity, the assumed boundary conditions are less sensitive.

(v) The present crisp results obtained from ADM are found to be in excellent agreement as compared to existing results.

(vi) In future work, for easier comprehension, the TFN is visualized. As a result, TFNs may be used to a variety of heat transfer challenges.

\section{Data Availability}

No data were used to perform this research.

\section{Conflicts of Interest}

The authors declare that they have no conflicts of interest.

\section{References}

[1] C. Truesdell and W. Noll, The nonlinear field's theories of mechanics, Springer, Heidelberg, Germany, third edition, 2004.

[2] K. R. Rajagopal, "On the stability of third grade fluids," Arch Mech, vol. 32, no. 6, pp. 867-875, 1980.

[3] K. R. Rajagopal, "Thermodynamics and stability of fluids of third grade," Pros R Soc lond A, vol. 339, pp. 351-377, 1980.

[4] A. M. Siddiqui, A. Zeb, Q. K. Ghori, and A. M. Benharbit, "Homotopy perturbation method for heat transfer flow of a third grade fluid between parallel plates," Chaos, Solitons \& Fractals, vol. 36, no. 1, pp. 182-192, 2008.

[5] T. Hayat, R. Ellahi, and F. M. Mahomed, "Exact solutions for thin film flow of a third grade fluid down an inclined plane," Chaos, Solitons \& Fractals, vol. 38, no. 5, pp. 1336-1341, 2008.

[6] M. Sajid and T. Hayat, "The application of homotopy analysis method to thin film flows of a third order fluid," Chaos, Solitons \& Fractals, vol. 38, no. 2, pp. 506-515, 2008.

[7] R. A. Shah, S. Islam, M. Zeb, and I. Ali, "Optimal homotopy asymptotic method for thin-film flows of a third-order fluid," J. of Adv. Research in Scientific Computing, vol. 2, pp. 1-14, 2011.

[8] A. M. Siddiqui, A. A. Farooq, T. Haroon, M. A. Rana, and B. S. Babcock, "Application of he's variational iterative method for solving thin film flow problem arising in nonnewtonian fluid mechanics," World Journal of Mechanics, vol. 02, no. 03, pp. 138-142, 2012.
[9] M. Yürüsoy, M. Pakdemirli, and B. S. Yilbas, "Perturbation solution for a third-grade fluid flowing between parallel plates," Proceedings of the Institution of Mechanical Engineers, Part C: Journal of Mechanical Engineering Science, vol. 222, no. 4, pp. 653-656, 2008.

[10] S. I. Natarov and C. P. Conrad, "The role of Poiseuille flow in creating depth-variation of asthenospheric shear," Geophysical Journal International, vol. 190, no. 3, pp. 1297-1310, 2012.

[11] T. Hayat, M. Khan, and M. Ayub, "Couette and Poiseuille flows of an Oldroyd 6-constant fluid with magnetic field," Journal of Mathematical Analysis and Applications, vol. 298, no. 1, pp. 225-244, 2004.

[12] T. Hayat, R. Naz, and M. Sajid, "On the homotopy solution for Poiseuille flow of a fourth grade fluid," Communications in Nonlinear Science and Numerical Simulation, vol. 15, no. 3, pp. 581-589, 2010.

[13] T. Chinyoka and O. D. Makinde, "Analysis of transient generalized Couette flow of a reactive variable viscosity thirdgrade liquid with asymmetric convective cooling," Math. Comput. Model, vol. 54, no. 1, pp. 160-174, 2011.

[14] G. Adomian, "A review of the decomposition method and some recent results for nonlinear equations," Math Comput Model, vol. 13, no. 7, pp. 287-99, 1992.

[15] G. Adomian, Solving frontier problems of physics: the decomposition method, Kluwer, Dordrecht, Netherland, 1994.

[16] Y. Cherruault and G. Adomian, "Decomposition methods: a new proof of convergence," Mathematical and Computer Modelling, vol. 18, no. 12, pp. 103-106, 1993.

[17] A. M. Siddiqui, M. Hameed, B. M. Siddiqui, and Q. K. Ghori, "Use of Adomian decomposition method in the study of parallel plate flow of a third grade fluid," Communications in Nonlinear Science and Numerical Simulation, vol. 15, no. 9, pp. 2388-2399, 2010.

[18] U. M. Pirzada and D. C. Vakaskar, "Solution of fuzzy heat equations using adomian decomposition method," Int. J. Adv. Appl. Math. Mech.vol. 3, no. 1, pp. 87-91, 2015.

[19] M. Paripour, E. Hajilou, and H. Heidari, "Application of Adomian decomposition method to solve hybrid fuzzy differential equations," J. of Taibah Uni. for Sci.vol. 57, pp. 1658-3655, 2014.

[20] A. M. Siddiqui, T. Haroon, S. Bhatti, and A. R. Ansari, "A comparison of the adomian and homotopy perturbation methods in solving the problem of squeezing flow between two circular plates," Mathematical Modelling and Analysis, vol. 15, no. 4, pp. 491-504, 2010.

[21] L. A. Zadeh, "Fuzzy Sets," Information and Control, vol. 8, no. 3, pp. 338-353, 1965.

[22] D. Dubois and H. Prade, "Operations on fuzzy numbers," International Journal of Systems Science, vol. 9, no. 6, pp. 613-626, 1978.

[23] S. Seikala, "On the fuzzy initial value problem," Fuzzy Sets and Systems, vol. 24, no. 3, pp. 319-330, 1987.

[24] O. Kaleva, "Fuzzy differential equations," Fuzzy Sets and Systems, vol. 24, no. 3, pp. 301-317, 1987.

[25] A. Kandel and W. J. Byatt, "Fuzzy differential equations," in Proceedings of International Conference Cybernetics and Society, vol. 1, pp. 1213-1216, Tokyo, Japan, November 1978.

[26] J. J. Buckley, T. Feuring, and Y. Hayashi, "Linear systems of first order ordinary differential equations: fuzzy initial conditions," Soft Computing - A Fusion of Foundations, Methodologies and Applications, vol. 6, no. 6, pp. 415-421, 2002. 
[27] J. J. Nieto, "The Cauchy problem for continuous fuzzy differential equations," Fuzzy Sets and Systems, vol. 102, no. 2, pp. 259-262, 1999.

[28] V. Lakshmikantham and R. N. Mohapatra, "Basic properties of solutions of fuzzy differential equations," Nonlinear Studies, vol. 8, pp. 113-124, 2000.

[29] J. Y. Park and H. K. Han, "Existence and uniqueness theorem for a solution of Fuzzy differential equations," International Journal of Mathematics and Mathematical Sciences, vol. 22, no. 2, pp. 271-279, 1999.

[30] M. S. Hashemi, J. Malekinagad, and H. R. Marasi, "Series solution of the system of fuzzy differential equations," Adv. in Fuzzy Syst, vol. 16, 2012.

[31] M. Mosleh, "Fuzzy neural network for solving a system of fuzzy differential equations," Applied Soft Computing, vol. 13, no. 8, pp. 3597-3607, 2013.

[32] N. Gasilov, S. E. Amrahov, and A. G. Fatullayev, "A geometric approach to solve fuzzy linear systems of differential equations," Appl. Math. Inf. Sci.vol. 5, no. 3, pp. 484-499, 2011.

[33] A. Khastan and J. J. Nieto, "A boundary value problem for second order fuzzy differential equations," Nonlinear Analysis: Theory, Methods \& Applications, vol. 72, no. 9-10, pp. 3583-3593, 2010.

[34] S. P. Mondal and T. K. Roy, "First order linear homogeneous ordinary differential equation in fuzzy environment based on laplace transform," J. of Fuzzy Set Valued Anal, vol. 1-18, 2013.

[35] H. Zarei, A. V. Kamyad, and A. A. Heydari, "Fuzzy modeling and control of hiv infection," Compu. Math. Meth. Medi.vol. 2017, Article ID 893474, 2012.

[36] S. P. Mondal, S. Banerjee, and T. K. Roy, "First order linear homogeneous ordinary differential equation in fuzzy environment," Int. J. Pure Appl. Sci. Tech, vol. 14, no. 1, pp. 16-26, 2013.

[37] M. Guo, X. Xue, and R. Li, "Impulsive functional differential inclusions and fuzzy population models," Fuzzy Sets and Systems, vol. 138, no. 3, pp. 601-615, 2003.

[38] L. C. Barros, R. C. Bassanezi, and P. A. Tonelli, "Fuzzy modelling in population dynamics," Ecological Modelling, vol. 128, no. 1, pp. 27-33, 2000.

[39] M. Z. Ahmad and B. De Baets, "A Predator-Prey Model with Fuzzy Initial Populations," in Proceedings of the Joint 2009 International Fuzzy Systems Association World Congress and 2009 European Society of Fuzzy Logic and Technology Conference, IFSA-EUSFLAT, Lisbon, Portugal, July 2009.

[40] J. Casasnovas and F. Rosselló, "Averaging fuzzy biopolymers," Fuzzy Sets and Systems, vol. 152, no. 1, pp. 139-158, 2005.

[41] G. L. Diniz, J. F. R. Fernandes, J. F. C. A. Meyer, and L. C. Barros, "A fuzzy cauchy problem modeling the decay of the biochemical oxygen demand in water,"vol. 1, pp. 512-516, in Proceedings Joint 9th IFSA World Congress and 20th NAFIPS International Conference, vol. 1, IEEE, Vancouver, BC, Canada, July 2001.

[42] A. Bencsik, B. Bede, J. Tar, and J. Fodor, "Fuzzy differential equations in modeling hydraulic differential servo cylinders," in In: Proceedings of the Third Romanian-Hungarian joint symposium on applied computational intelligence (SACI), Timisoara, Romania, 2006.

[43] B. Bede, I. J. Rudas, and J. Fodor, "Friction model by fuzzy differential equations," vol. 4529, pp. 23-32, in Proceedings of the Foundations of Fuzzy Logic and Soft Computing, 12th International Fuzzy Systems Association World Congress, IFSA 2007, vol. 4529, pp. 23-32, Springer-Verlag, Cancun, Mexico, June 2007.
[44] M. S. El Naschie, "From experimental quantum optics to quantum gravity via a fuzzy Kähler manifold," Chaos, Solitons \& Fractals, vol. 25, no. 5, pp. 969-977, 2005.

[45] T. Allahviranloo and S. Salahshour, "Applications of fuzzy Laplace transforms," Soft Computing, vol. 17, no. 1, pp. 145-158, 2013.

[46] M. Oberguggenberger and S. Pittschmann, "Differential equations with fuzzy parameters," Math. Mod. Syst, vol. 5, pp. 181-202, 1999.

[47] S. Hajighasemi, T. Allahviranloo, M. Khezerloo, M. Khorasany, and S. Salahshour, "Existence and uniqueness of solutions of fuzzy Volterra integro-differential equations," Communications in Computer and Information Science, vol. 81, pp. 491-500, 2010.

[48] U. Biswal, S. Chakraverty, and B. K. Ojha, "Natural convection of nanofluid flow between two vertical flat plates with imprecise parameter," Coupled Systems Mechanics, vol. 9, no. 3, pp. 219-235, 2020.

[49] G. Borah, P. Dutta, and G. C. Hazarika, "Numerical study on second-grade fluid flow problems using analysis of fractional derivatives under fuzzy environment," Soft Computing Techniques and Applications. Advances in Intelligent Systems and Computing, vol. 1248, 2021.

[50] M. Nadeem, I. Siddique, F. Jarad, and R. N. Jamil, "Numerical study of mhd third-grade fluid flow through an inclined channel with ohmic heating under fuzzy environment," Mathematical Problems in Engineering, vol. 2021, Article ID 9137479, 17 pages, 2021.

[51] U. Biswal, S. Chakraverty, B. K. Ojha, and A. K. Hussein, "Study of Jeffery-Hamel flow problem for nanofluid with fuzzy volume fraction using double parametric based Adomian decomposition method," International Communications in Heat and Mass Transfer, vol. 126, Article ID 105435, 2021.

[52] E. 1. Allaoui, S. Melliani, and L. S. Chadli, "A mathematical fuzzy model to giving up smoking," in Proceedings of the IEEE 6th Inter. Conference on Optimization and Appication, pp. 1-6, ICOA, Beni Mellal, Morocco, April 2020.

[53] R. M. Zulqarnain, X. L. Xin, I. Siddique, W. Asghar Khan, and M. A. Yousif, "TOPSIS method based on correlation coefficient under pythagorean fuzzy soft environment and its application towards green supply chain management," Sustainability, vol. 13, no. 4, 1642 pages, 2021.

[54] R. M. Zulqarnain, I. Siddique, R. Ali, F. Jarad, A. Samad, and T. Abdeljawad, "Neutrosophic hypersoft matrices with application to solve multiattributive decision-making problems," Complexity, vol. 2021, Article ID 5589874, 17 pages, 2021.

[55] R. M. Zulqarnain, I. Siddique, R. Ali, D. Pamucar, D. Marinkovic, and D. Bozanic, "Robust aggregation operators for intuitionistic fuzzy hypersoft set with their application to solve MCDM problem," Entropy, vol. 23, no. 6, 688 pages, 2021.

[56] R. M. Zulqarnain, I. Saddique, F. Jarad, R. Ali, and T. Abdeljawad, "Development of TOPSIS technique under pythagorean fuzzy hypersoft environment based on correlation coefficient and its application towards the selection of antivirus mask in COVID-19 pandemic," Complexity, vol. 2021, Article ID 6634991, 27 pages, 2021.

[57] I. Siddique, R. M. Zulqarnain, R. Ali, F. Jarad, and A. Iampan, "Multicriteria decision-making approach for aggregation operators of pythagorean fuzzy hypersoft sets," Computational Intelligence and Neuroscience, vol. 2021, pp. 1687-5265, Article ID 2036506, 2021. 
[58] M. Nadeem, A. Elmoasry, I. Siddique et al., "Study of triangular fuzzy hybrid nanofluids on the natural convection flow and heat transfer between two vertical plates," Computational Intelligence and Neuroscience, vol. 2021, Article ID 3678335, 15 pages, 2021.

[59] I. Siddique, R. M. Zulqarnain, M. Nadeem, and F. Jarad, "Numerical simulation of mhd couette flow of a fuzzy nanofluid through an inclined channel with thermal radiation effect," Computational Intelligence and Neuroscience, vol. 2021, Article ID 6608684, 16 pages, 2021. 